\title{
Nonequilibrium critical dynamics of the two-dimensional Ashkin-Teller model at the Baxter line
}

\author{
H. A. Fernandes, ${ }^{1}$ R. da Silva, ${ }^{2}$ A. A. Caparica, ${ }^{3}$ and J. R. Drugowich de Felício ${ }^{4}$ \\ ${ }^{1}$ Universidade Federal de Goiás - UFG, Campus Jataí,, Jataí-GO, 78000-000, Brazil \\ ${ }^{2}$ Instituto de Física, Universidade Federal do Rio Grande do Sul, UFRGS, Porto Alegre - RS, 91501-970, Brazil \\ ${ }^{3}$ Instituto de Física, Universidade Federal de Goiás, Goiânia-GO, 74.690-900, Brazil \\ ${ }^{4}$ Departamento de Física, Universidade de São Paulo, Ribeirão Preto-SP, 14040-901, Brazil
}

(Received 18 December 2016; published 5 April 2017)

\begin{abstract}
We investigate the short-time universal behavior of the two-dimensional Ashkin-Teller model at the Baxter line by performing time-dependent Monte Carlo simulations. First, as preparatory results, we obtain the critical parameters by searching the optimal power-law decay of the magnetization. Thus, the dynamic critical exponents $\theta_{m}$ and $\theta_{p}$, related to the magnetic and electric order parameters, as well as the persistence exponent $\theta_{g}$, are estimated using heat-bath Monte Carlo simulations. In addition, we estimate the dynamic exponent $z$ and the static critical exponents $\beta$ and $v$ for both order parameters. We propose a refined method to estimate the static exponents that considers two different averages: one that combines an internal average using several seeds with another, which is taken over temporal variations in the power laws. Moreover, we also performed the bootstrapping method for a complementary analysis. Our results show that the ratio $\beta / v$ exhibits universal behavior along the critical line corroborating the conjecture for both magnetization and polarization.
\end{abstract}

DOI: 10.1103/PhysRevE.95.042105

\section{INTRODUCTION}

In 1971 Baxter [1] calculated the free energy of the symmetric eight-vertex model and found out for the first time a continuous dependence of the critical exponents on the coupling coefficients of the model. This result seemed, in principle, to contradict the universality hypothesis [2-4], which suggests that the critical exponents should be constant and a variation would be possible only in the case of a change in the symmetry. Despite this apparent contradiction, Kadanoff and Wegner [5] and Wu [6] showed independently a connection between the continuous variation of those exponents and the presence of a marginal operator in the Hamiltonian by demonstrating the equivalence of this model with an Ising model in a square lattice without field. In this formulation, besides the interactions between next-nearest neighbors, there is still a four-body interaction and the Hamiltonian is written as [7]:

$$
\begin{aligned}
\beta \mathcal{H}_{8 V}= & -J_{1} \sum_{i, j=1}^{L} \sigma_{i, j+1} \sigma_{i+1, j}-J_{2} \sum_{i, j=1}^{L} \sigma_{i j} \sigma_{i+1, j+1} \\
& -\lambda \sum_{i, j=1}^{L} \sigma_{i j} \sigma_{i, j+1} \sigma_{i+1, j} \sigma_{i+1, j+1},
\end{aligned}
$$

where $\sigma_{i j}= \pm 1$ is the Ising spin at the site $(i, j)$ of the lattice, $\beta=\left(k_{B} T\right)^{-1}, k_{B}$ and $T$ being, respectively, the Boltzmann constant and the temperature of the system. The sums run over all spins and periodic boundary conditions are assumed: $\sigma_{L+1, j}=\sigma_{1, j}$ and $\sigma_{i, L+1}=\sigma_{i, 1}$. The spins are coupled by the coefficient $J_{1}$ in one direction and by $J_{2}$ in the other one and the coefficient $\lambda$ couples four spins.

The symmetric eight-vertex model, also known as the Baxter model, has only one critical line, where $J_{1}=J_{2}=J$. This line is given by the equation [7]

$$
\exp (-2 \lambda)=\sinh (2 J) \text {. }
$$

Besides the eight-vertex model there are other models that exhibit nonuniversality, e.g., the Ising model with competing interactions [8] and the Ashkin-Teller model [9]. The latter was introduced in 1943 to describe a four-component system with nearest-neighbors interactions, displaced on a twodimensional lattice. Soon after Baxter's work, Fan [10] showed that the Ashkin-Teller (AT) model could be represented by two superposed Ising systems and coupled by a four-body interaction coefficient.

In this representation the Hamiltonian for the AT model is given by two-species model:

$$
\begin{aligned}
\beta \mathcal{H}_{A T}= & -K_{1} \sum_{i, j=1}^{L} \sigma_{i, j}\left(\sigma_{i, j+1}+\sigma_{i+1, j}\right) \\
& -K_{2} \sum_{i, j=1}^{L} \mu_{i, j}\left(\mu_{i, j+1}+\mu_{i+1, j}\right) \\
& -K_{4} \sum_{i, j=1}^{L} \sigma_{i, j} \mu_{i, j}\left(\sigma_{i+1, j} \mu_{i+1, j}+\sigma_{i, j+1} \mu_{i, j+1}\right)
\end{aligned}
$$

where $\sigma_{i, j}= \pm 1\left(\mu_{i, j}= \pm 1\right)$ is the Ising spin at the site $(i, j)$ of the sublattice $\sigma(\mu), K_{1}\left(K_{2}\right)$ is the coupling coefficient of the spin variable $\sigma_{i, j}\left(\mu_{i, j}\right)$, and $K_{4}$ is the four-body coefficient, which couples the two Ising systems. The sums run over all spins and periodic boundary conditions are assumed: $\sigma(\mu)_{L+1, j}=\sigma(\mu)_{1, j}$ and $\sigma(\mu)_{i, L+1}=\sigma(\mu)_{i, 1}$.

Wegner [11] showed that by carrying out a duality transformation in one of the lattices ( $\mu$, for example), one can map the AT model into a staggered eight-vertex model. This alternation does not disappear even for the isotropic model $\left(K_{1}=K_{2}=K\right)$ except at the self-dual line

$$
\exp \left(-2 K_{4}\right)=\sinh (2 K)
$$

where the AT model becomes equivalent to an isotropic eight-vertex model with four-spin coupling constant $(\lambda)$ given 
by

$$
\tanh (2 \lambda)=\frac{\tanh \left(2 K_{4}\right)}{\tanh \left(2 K_{4}\right)-1},
$$

which is critical if $K_{4}<\frac{1}{4} \ln 3$ [7], with critical exponents related by [12-14]:

$$
2-\frac{1}{v_{A T}}=\frac{1}{\left(2-1 / \nu_{8 V}\right)},
$$

where

$$
\frac{1}{v_{8 V}}=1-\frac{2}{\pi} \sin ^{-1}[\tanh (2 \lambda)] .
$$

In the Ashkin-Teller model, besides the magnetization $M$ of each sublattice, another order parameter is present: the polarization $P$. These order parameters are defined as

$$
\begin{aligned}
M_{\sigma} & =\frac{1}{L^{2}}\left\langle\sum_{i, j=1}^{L} \sigma_{i, j}\right\rangle, \quad M_{\mu}=\frac{1}{L^{2}}\left\langle\sum_{i, j=1}^{L} \mu_{i, j}\right\rangle, \\
P & =\frac{1}{L^{2}}\left\langle\sum_{i, j=1}^{L} \sigma_{i, j} \mu_{i, j}\right\rangle,
\end{aligned}
$$

where $\langle\cdot\rangle$ denotes the ensemble average:

$$
\langle(\cdot)\rangle=\frac{1}{Z} \sum_{\left\{\sigma_{i, j}, \mu_{i, j}\right\}}(\cdot) \exp \left[-\beta \mathcal{H}_{A T}\left(\left\{\sigma_{i, j}, \mu_{i, j}\right\}_{i, j=1}^{L}\right)\right]
$$

with $Z=\sum_{\left\{\sigma_{i, j}, \mu_{i, j}\right\}} \exp \left[-\beta \mathcal{H}_{A T}\left(\left\{\sigma_{i, j}, \mu_{i, j}\right\}_{i, j=1}^{L}\right)\right]$.

However, as we are dealing with the isotropic version of the model, the spins of each sublattice are symmetric and, in this case, their magnetizations will have the same behavior. Then the net result is that the number of samples for the magnetization is doubled. Henceforth, we consider only two order parameters: the magnetization $(M)$ that includes both sublattices, and the polarization $(P)$.

The purpose of this paper is to study the dynamic critical behavior of the Ashkin-Teller model to obtain the dynamic exponents $\theta_{g}, \theta$, and $z$, as well as the static exponents $\beta$ and $v$ for both order parameters. To reach our goal, we carry out short-time Monte Carlo (MC) simulations in the two-dimensional isotropic AT model. The paper is organized as follows. In the next section, we briefly present the nonequilibrium technique as well as the scaling relations used in this work. In Sec. III, we find out the critical exponents of the Ashkin-Teller model. Finally, in Sec. IV we present our conclusions.

\section{CRITICAL DYNAMICS FOR THE MODEL}

Until a few years ago, it was commonly believed that no universal behavior could be found in systems during the initial stage of the relaxation process. As a result, critical properties of those systems, such as transition temperatures and critical exponents, were obtained only in equilibrium. The numerical calculation of such values was not a simple task, due to the severe critical slowing down that takes place in the vicinity of the criticality. Many efforts have been made to circumvent this difficulty, for instance, the cluster algorithm $[15,16]$ has proven to be very efficient in the study of static properties of systems. Nevertheless, in that case the original dynamic class of universality is violated, leading to normally small values for the dynamic critical exponents. Another way to avoid problems with the critical slowing down was proposed by Janssen et al. [17] and Huse [18]. Using renormalization group techniques and numerical calculation, respectively, they showed that the critical relaxation of a system initially at very high temperature exhibits universality and scaling behavior even in the initial steps of evolution. The so-called short-time regime became, therefore, an important method in the study of phase transitions and critical phenomena.

The dynamic scaling relation obtained by Janssen $e t$ al. for the $k$ th moment of the magnetization, extended to systems of finite size $[19,20]$, is written as

$$
\overline{M^{k}}\left(t, \tau, L, m_{0}\right)=b^{-\frac{k \beta}{v}} \overline{M^{k}}\left(b^{-z} t, b^{\frac{1}{v}} \tau, b^{-1} L, b^{x_{0}} m_{0}\right) .
$$

Here $t$ is the time evolution, $b$ is an arbitrary spatial rescaling factor, $\tau=\left(T-T_{c}\right) / T_{c}$ is the reduced temperature, and $L$ is the linear size of the square lattice. This evolution is governed by a new dynamic exponent $\theta$ independent of the well-known static critical exponents and the dynamic exponent $z$. This new exponent characterizes the so-called critical initial slip, the anomalous behavior of the magnetization when the system is quenched to the critical temperature $T_{c}$. In addition, a new critical exponent $x_{0}$, which represents the anomalous dimension of the initial magnetization $m_{0}$, is introduced to describe the dependence of the scaling behavior on the initial conditions. This exponent is related to $\theta$ as $x_{0}=\theta z+\beta / \nu$.

From Eq. (9), the scaling relations for the $k$ th moment of the magnetization and polarization of the Ashkin-Teller model are given, respectively, by

$$
\overline{M^{k}}\left(t, \tau, L, m_{0}\right)=b^{-\frac{k \beta m}{v}} \overline{M^{k}}\left(b^{-z_{m}} t, b^{\frac{1}{v}} \tau, b^{-1} L, b^{x_{m}} m_{0}\right)
$$

and

$$
\overline{P^{k}}\left(t, \tau, L, p_{0}\right)=b^{-\frac{k \beta_{p}}{v}} \overline{P^{k}}\left(b^{-z_{p}} t, b^{\frac{1}{v}} \tau, b^{-1} L, b^{x_{p}} p_{0}\right),
$$

where $p_{0}$ is the initial polarization of the system. Here, differently from $\langle O\rangle$, the average $\bar{O}$ describes an average over different random evolutions and initial conditions of the system.

In this work the dynamic critical exponents $\theta_{m}$ and $\theta_{p}$ are obtained through two different approaches. In the first one, we consider the time correlation of the order parameters [21] given by

$$
Q_{M}(t)=\overline{M(0) M(t)} \sim t^{\theta_{m}}
$$

and

$$
Q_{P}(t)=\overline{P(0) P(t)} \sim t^{\theta_{p}} .
$$

This approach takes into account different initial random configurations of spins for the different time evolutions, the only requirement being that $\overline{M(0)}=\overline{m_{0}} \approx 0$ [and similarly $\overline{P(0)}=\overline{p_{0}} \approx 0$ ]. The second approach considers a fixed and small initial magnetization and polarization $\left(m_{0}\right.$ and $\left.p_{0}\right)$ for the different time evolutions, which leads to the following scaling forms:

$$
\bar{M}(t) \sim m_{0} t^{\theta_{m}}
$$


and

$$
\bar{P}(t) \sim p_{0} t^{\theta_{p}}
$$

In order to observe such power-law behaviors we can look into some details of the scaling relations. However, as the two order parameters follow similar behaviors, we will consider only the magnetization and extend the results to the polarization. In a regime of small initial magnetization and soon after a finite time scaling $b=t^{1 / z}$, at the critical temperature $(\tau=0)$ and for large systems $(L \rightarrow \infty)$, Eq. (9) can be written as

$$
\bar{M}\left(t, m_{0}\right)=t^{-\beta /(v z)} \bar{M}\left(1, t^{x_{0} / z} m_{0}\right) .
$$

By calling $x=t^{x_{0} / z} m_{0}$, an expansion of the averaged magnetization around $x=0$ results in

$$
\bar{M}(1, x)=\bar{M}(1,0)+\left.\partial_{x} \bar{M}\right|_{x=0} x+O\left(x^{2}\right) .
$$

By construction $\bar{M}(1,0)=0$ and, since $u=t^{x_{0} / z} m_{0} \ll 1$, one can discard quadratic terms resulting in $\langle M\rangle_{m_{0}} \sim m_{0} t^{\theta}$, with $\theta=\left(x_{0}-\beta / v\right) / z$. This anomalous behavior of the initial magnetization is valid only for $t<t_{\max }$, where $t_{\max } \sim$ $m_{0}^{-z / x_{0}}$. Here it is important to make clear an interesting point. Let us consider the time correlation function $Q_{M}(t)=$ $\frac{1}{L^{d}} \overline{\sum_{i} \sigma_{i}(0) \sum_{j} \sigma_{j}(t)}$ as an example (where $d$ is the dimension of the system). We can expand this function obtaining

$$
Q_{M}(t)=\frac{1}{L^{d}} \overline{\sum_{i=1}^{L^{d}} \sigma_{i}(0) \sigma_{i}(t)}+\frac{1}{L^{d}} \overline{\sum_{i \neq j} \sigma_{i}(0) \sigma_{j}(t)},
$$

where the term $A(t)=\frac{1}{L^{d}} \overline{\sum_{i} \sigma_{i}(0) \sigma_{i}(t)}$ is known as the spinspin autocorrelation function. When the spins are randomly distributed on the lattice sites with $m_{0}$ exactly equal to 0 in all time evolutions used to compose the average, we must expect $A(t) \sim t^{-\Lambda_{m}}$, where $\Lambda_{m}$ (the time autocorrelation exponent) is related to $\theta_{m}$ (the exponent of the time correlation of the total magnetization) according to a closed form (see, for example, Refs. [20,22]):

$$
\Lambda_{m}=d / z_{m}-\theta_{m}
$$

Another dynamic critical exponent is obtained far from equilibrium by following the behavior of the global persistence probability $G(t)$ [22], the probability of the order parameter does not change its sign up to the time $t$. For the magnetization (and polarization) at the critical temperature, it decays, respectively, as

$$
G_{M}(t) \sim t^{-\theta_{g m}}
$$

and

$$
G_{P}(t) \sim t^{-\theta_{g_{p}}},
$$

where the exponents $\theta_{g_{m}}$ and $\theta_{g_{p}}$ are the global persistence exponents of the magnetization and polarization.

As pointed out in Ref. [22] and shown in several works [23-36], the global persistence exponent is an independent critical index and is closely related to the non-Markovian character of the process. If the magnetization is a Markovian random variable, which is not the case in the present work, this exponent should obey the equation

$$
\theta_{g_{m}} z_{m}=-\theta_{m} z_{m}+\frac{d}{z_{m}}-\frac{\beta_{m}}{v_{m}},
$$

or simply $\theta_{g_{m}} z_{m}=\left(1-z_{m}\right) \theta_{m}+\Lambda_{m}-\frac{\beta_{m}}{v_{m}}$.

The dynamic critical exponents $z_{m}$ and $z_{p}$ are obtained using the ratios [37]

$$
F_{2_{M}}(t)=\frac{\overline{M(t)^{2}}{ }_{m_{0}=0}}{\overline{M(t)}_{m_{0}=1}^{2}} \sim t^{d / z_{m}}
$$

and

$$
F_{2_{P}}(t)=\frac{\overline{P(t)^{2}}{ }_{p_{0}=0}}{{\overline{P(t)_{p_{0}=1}^{2}}}_{p^{2}}} \sim t^{d / z_{p}},
$$

where the average is over different samples with initial states $m_{0}$ and $p_{0}$, respectively.

The first moment of the magnetization in Eq. (23) (in the denominator) is obtained by considering the power-law decay obtained by considering ordered initial state $\left(m_{0}=1\right)$ in the scaling relation given by Eq. (16). Since the system has no dependence on initial conditions, one has

$$
\bar{M}_{m_{0=1}}(t) \sim t^{-\frac{\beta_{m}}{v_{m} z_{m}}} .
$$

The same analysis can be done for the polarization, obtaining

$$
\bar{P}_{p_{0}=1}(t) \sim t^{-\frac{\beta_{p}}{v_{p} p}}
$$

On the other hand, the second moment of the magnetization in Eq. (23) (the numerator) can be written as

$$
\overline{M_{m_{0}=0}^{2}}=\frac{1}{L^{2 d}} \sum_{i=1}^{L^{d}} \overline{\sigma_{i}^{2}}+\frac{1}{L^{2 d}} \sum_{i \neq j}^{L^{d}} \overline{\sigma_{i} \sigma_{j}} \approx L^{-d}
$$

for a fixed $t$. By taking into account $k=2$ in Eq. (10) with $b=t^{1 / z_{m}}$ and considering that the spin-spin correlation $\overline{\sigma_{i} \sigma_{j}}$ is negligible for $m_{0}=0$, we obtain

$$
\begin{aligned}
\overline{M_{m_{0}=0}^{2}}(t, L) & \approx t^{\frac{-2 \beta_{m}}{v_{m} z_{m}}} \overline{M_{m_{0}=0}^{2}}\left(1, t^{-1 / z} L\right) \\
& =t^{\frac{-2 \beta_{m}}{v_{m} z_{m}}}\left(t^{-1 / z} L\right)^{-d} \\
& \sim t^{\left(d-\frac{2 \beta_{m}}{v_{m}}\right) / z_{m}}
\end{aligned}
$$

for the second moment of the magnetization and, similarly,

$$
\overline{P_{p_{0}=0}^{2}}(t, L) \sim t^{\left(d-\frac{2 \beta_{p}}{v_{p}}\right) / z_{p}}
$$

for the second moment of the polarization. Therefore, the power laws given by Eqs. (23) and (24) can be easily verified.

This approximation proved to be very efficient in estimating the exponent $z$, according to results for the Ising model, the three-state and four-state Potts models [37], the tricritical point of the Blume-Capel model [38], metamagnetic model [39], ANNNI model [40], spin models based on generalized Tsallis statistics [41], Z5 model [42], the Baxter-Wu model [43], the double-exchange model [44], Heisenberg model [34], protein folding models [45], even models without defined Hamiltonian (see, for example, Refs. [27,46,47]).

The static exponents must be obtained via other power laws. When $L \rightarrow \infty$, one has $\bar{M}(t, \tau)=b^{-k \beta / \nu} \bar{M}\left(b^{-z} t, b^{1 / v} \tau\right)$. 
By scaling $b^{-z} t=1$, we have $\bar{M}(t, \tau)=t^{-\beta /(v z)} f\left(t^{1 /(v z)} \tau\right)$ where $f(x)=\bar{M}(1, x)$ and so $\partial \ln \bar{M}(t, \tau) / \partial \tau=\frac{1}{\langle M\rangle} \frac{\partial}{\partial \tau} \bar{M}=$ $t^{1 /(v z)} f\left(t^{1 /(v z)} \tau\right)$. Therefore, we have

$$
D_{M}(t)=\left.\frac{\partial \ln \bar{M}}{\partial \tau}\right|_{\tau=0}=f_{0} \cdot t^{1 /\left(v_{m} z_{m}\right)} \sim t^{\phi_{m}},
$$

where $f_{0}=f(0)$ is a constant and $\phi_{m}=1 /\left(v_{m} z_{m}\right)$. Since we have already estimated the exponent $z_{m}$ [Eq. (23)], we are able to obtain $v_{m}$. With these two exponents in hand, we can obtain $\beta_{m}$ by estimating the exponent $\mu_{m}=\beta_{m} /\left(v_{m} z_{m}\right)$ from Eq. (25). By changing $M$ by $P$, we have

$$
D_{P}(t)=\left.\frac{\partial \ln \bar{P}}{\partial \tau}\right|_{\tau=0} \sim t^{\phi_{p}}
$$

with $\phi_{p}=1 /\left(v_{p} z_{p}\right)$, where the exponents $v_{p}$ and $\beta_{p}$ are obtained by following the same procedures adopted for the magnetization.

\section{SOME DETAILS ABOUT HEAT-BATH MONTE CARLO SIMULATIONS}

In this section, we describe with some details how the heatbath Monte Carlo simulations are carried out in our work to evolve the spins. The interesting point here is that the transition occurs for the pair of spins $\left(\sigma_{i, j}, \mu_{i, j}\right)$ and not for single spins since we have coupled lattices. Moreover, this transition does not depend on current spin. So, the transition probabilities of each possible pair: $(+,+),(-,+),(+,-)$, and $(-,-)$ are calculated by

$$
p\left[\cdot \rightarrow\left(\sigma_{i, j}, \mu_{i, j}\right)\right]=\frac{1}{S} \exp \left[-E\left(\sigma_{i, j}, \mu_{i, j}\right)\right]
$$

with $S=e^{-E(+,+)}+e^{-E(-,+)}+e^{-E(+,-)}+e^{-E(-,-)}$ and

$$
\begin{aligned}
& E(+,+)=-K\left(\Delta_{i, j}+\Psi_{i, j}\right)-K_{4} \Phi_{i, j}, \\
& E(-,+)=-K\left(\Psi_{i, j}-\Delta_{i, j}\right)+K_{4} \Phi_{i, j}, \\
& E(+,-)=-K\left(\Delta_{i, j}-\Psi_{i, j}\right)+K_{4} \Phi_{i, j}, \\
& E(-,-)=K\left(\Delta_{i, j}+\Psi_{i, j}\right)-K_{4} \Phi_{i, j},
\end{aligned}
$$

where

$$
\begin{aligned}
& \Delta_{i, j}=\sigma_{i+1, j}+\sigma_{i-1, j}+\sigma_{i, j+1}+\sigma_{i, j-1}, \\
& \Psi_{i, j}=\mu_{i+1, j}+\mu_{i-1, j}+\mu_{i, j+1}+\mu_{i, j-1}
\end{aligned}
$$

and

$$
\begin{aligned}
\Phi_{i j}= & \sigma_{i+1, j} \mu_{i+1, j}+\sigma_{i-1, j} \mu_{i-1, j}+\sigma_{i, j+1} \mu_{i, j+1} \\
& +\sigma_{i, j-1} \mu_{i, j-1} .
\end{aligned}
$$

For the AT model the relevant quantities correspond to time-dependent magnetization, polarization, as well as their superior moments, here represented by a general symbol $O$ defined via our MC simulations as an average over all $L^{2}$ spins and over the different $N_{\text {run }}$ runs (the number of different time evolutions):

$$
\bar{O}(t)=\frac{1}{N_{\text {run }} L^{2}} \sum_{k=1}^{N_{\text {run }}} \sum_{i, j=1}^{L} O_{i, j, k}(t),
$$

where the index $k=1, \ldots, N_{\text {run }}$ denotes the corresponding run of each simulation. The ordered state is ferromagnetic, with all (or most of) the spins pointing either up or down.

As discussed in the previous section, the lattice's initial condition to be simulated in our study depends on the scaling relation as follows.

(i) Equations (12) and (13): To obtain such power laws, the averages are obtained from a set of runs with initially random configurations allowing the direct calculation of the dynamic exponents $\theta_{m}$ and $\theta_{p}$. Here, the only requirement is that $\left\langle m_{0}\right\rangle=\left\langle p_{0}\right\rangle=0$. Unfortunately, the huge fluctuations for $P(t)$, even for $N_{\text {run }}=3 \times 10^{5}$ runs, prevented us from estimating $\theta_{p}$ through this method.

(ii) Equations (14) and (15): In order to obtain the same exponents $\theta_{m}$ and $\theta_{p}$ we use these alternative equations. However, in this case, a careful preparation of the initial order parameters $\left(m_{0}\right.$ and $\left.p_{0}\right)$ is needed, besides the limit procedures $m_{0} \rightarrow 0$ and $p_{0} \rightarrow 0$. Here we used $N_{\text {run }}=10^{5}$ runs.

(iii) Equations (25) and (26): In order to perform the simulations to obtain the exponents by these power laws, we used ordered initial states, which means $m_{0}=1$ and $p_{0}=1$. In this particular case the simulations do not present sensitive fluctuations and for all cases we used $N_{\text {run }}=4000$ runs.

(iv) Equations (27) and (28): When computing the second moment of the magnetization or polarization, we used $m_{0}=0$ [half (randomly chosen) of the spins up and the other half of the spins down] and $N_{\text {run }}=4000$ runs.

(v) Equation (29): When dealing with Monte Carlo simulations, the partial derivative is approximated in first order by the difference

$$
\left.\frac{\left.\partial \ln \bar{M}(t, \tau)\right|_{m_{0}=1}}{\partial \tau}\right|_{\tau=0} \approx \frac{1}{2 \varepsilon} \ln \left[\frac{\left.\bar{M}\left(t, T_{c}+\varepsilon\right)\right|_{m_{0}=1}}{\left.\bar{M}\left(t, T_{c}-\varepsilon\right)\right|_{m_{0}=1}}\right],
$$

where $\varepsilon \ll 1$. It is clear from Eq. (32) that two independent simulations are necessary to obtain the exponent $1 / v z$ : one of them evolves at the temperature $T_{c}+\varepsilon$, and the other one evolves at $T_{c}-\varepsilon$. Here we used $N_{\text {run }}=4000$ runs for $\left.\bar{M}\left(t, T_{c}+\varepsilon\right)\right|_{m_{0}=1}$ and $N_{\text {run }}=4000$ runs for $\left.\bar{M}\left(t, T_{c}-\varepsilon\right)\right|_{m_{0}=1}$ since we start from ordered initial states.

It is important to mention that the number of runs used in this work is equal to or larger than $N_{\text {run }}$ used in other successful works (see, for example, Refs. [33] and [40]).

\section{LOCALIZATION OF CRITICAL POINTS: POWER LAW OPTIMIZATION}

In this section we performed some initial simulations to give more knowledge about the criticality of the AT model. The theoretical predictions of the critical line are described by

$$
K_{4}(K)=-\frac{1}{2} \ln [\sinh (2 K)] .
$$

Therefore, let us consider a particular critical point of this curve, denoted by $\left[K^{(c)}, K_{4}^{(c)}\right]$, which corresponds to a particular critical coefficient $J_{c}$ of the Baxter model, such that $\lambda_{c}=-\frac{1}{2} \ln \left[\sinh \left(2 J_{c}\right)\right]$. Hence, we obtain $K_{4}^{(c)}=$ $\frac{1}{2} \tanh ^{-1}\left(\frac{\tanh \left(2 \lambda_{c}\right)}{\tanh \left(2 \lambda_{c}\right)-1}\right)$ and $K^{(c)}=\frac{1}{2} \sinh ^{-1}\left[\exp \left(-2 K_{4}^{(c)}\right)\right]$. 
TABLE I. The five points in the self-dual critical line.

\begin{tabular}{lcccc}
\hline \hline Critical point $(\mathrm{CP})$ & $J$ & $\lambda$ & $K$ & $K_{4}$ \\
\hline P1 & 0.4 & 0.059332097 & 0.489889651 & -0.067369092 \\
Ising Model & $0.5 \ln (1+\sqrt{2})$ & 0 & $0.5 \ln (1+\sqrt{2})$ & 0 \\
P2 & 0.5 & -0.080719681 & 0.393334281 & 0.069427372 \\
P3 & 0.596439479 & -0.20159986 & 0.347625611 & 0.142089631 \\
FSP Model & $+\infty$ & $-\infty$ & $(\ln 3) / 4$ & $(\ln 3) / 4$ \\
\hline \hline
\end{tabular}

The critical exponents at the equilibrium state for the AT model are well known and given by

$$
\begin{aligned}
v & =v_{m}=v_{p}=\frac{2-y}{3-2 y}, \\
\beta_{m} & =\frac{2-y}{24-16 y} \quad \beta_{p}=\frac{1}{12-8 y},
\end{aligned}
$$

where the parameter $y$ belongs to the interval $[0,4 / 3]$ and is given by

$$
y=\frac{2}{\pi} \cos ^{-1}\left[\frac{1}{2}\left(e^{4 K_{4}}-1\right)\right] .
$$

In Table I, we collected the five critical points considered in this work $(J)$ as well as the four-body coupling constants $(\lambda)$ at the critical line of the Baxter model. The corresponding coefficients $K$ and $K_{4}$ for the Ashkin-Teller model, calculated from Eqs. (2), (4), and (5), are also shown in this table. We choose the points, $\mathrm{P} 1: J=0.4$, which corresponds to $y \simeq 1.0754$; the Ising point, which corresponds to $J=\frac{1}{2} \ln (1+\sqrt{2})$ or $y=1$; P2: $J=0.5$ corresponding to $y \simeq 0.8977$; and the point P3, which has the same thermal exponent $(v=5 / 6)$ of three-state Potts model, corresponding to $J \simeq 0.596$ which corresponds to $y=3 / 4$. This point was reported in Ref. [53] as belonging to the universality class of the three-state Potts model. However, in an erratum, the author has fixed this mistake. For example we can observe the difference for the exponent $\beta=1 / 9$ for the three-state Potts model while for the point P3 we have $\beta_{m}=5 / 48$. The last point in Table I is the four-state Potts (FSP) point corresponding to $J \rightarrow+\infty$ (or $y=0$ ).

In Fig. 1 we present the critical line of the AT model and illustrate the points to be considered in this study, as well as the perpendicular lines for each point. In order to find these perpendicular lines, we first focus on the tangent lines to the curve $K_{4}(K)=-\frac{1}{2} \ln [\sinh (2 K)]$ passing through the critical points $\left[K^{(c)}, K_{4}^{(c)}\right]$. These tangent lines are written as

$$
K_{4}^{\|}=-\operatorname{coth}\left(2 K_{c}\right)\left(K-K_{c}\right)-\frac{1}{2} \ln \left[\sinh \left(2 K_{c}\right)\right],
$$

and the perpendicular line to this tangent line, can be written as

$$
K_{4}^{\perp}=\tanh \left(2 K_{c}\right)\left(K-K_{c}\right)-\frac{1}{2} \ln \left[\sinh \left(2 K_{c}\right)\right] .
$$

Our initial plan was to study the phase transition points of the AT model via time-dependent MC simulations by estimating the best $K$ given as input the parameter $K^{(\min )}$ (inicial value) and run simulations for different values of $K$ according to a resolution $\Delta K$.

We performed this task for the five points shown in Fig. 1 by taking into account only the magnetization and the analysis was carried out by using an approach developed in Ref. [41] in the context of generalized statistics. This tool had also been applied successfully to study multicritical points, for example, tricritical points [39] and Lifshitz point of the ANNNI model [40], Z5 model [42], and also in models without defined Hamiltonian [48].

Since at criticality it is expected that the order parameter obeys the power-law behavior of Eq. (25), for each value $K=K^{(\mathrm{min})}+i \Delta K$, with $i=1, \ldots, n$, where $n=$ $\left\lfloor\left(K^{(\max )}-K^{(\min )}\right) / \Delta K\right\rfloor$, we performed MC simulations and calculated the coefficient of determination, which is given by

$$
r=\frac{\left.\sum_{t=1}^{N_{M C}} \overline{\overline{(\ln \overline{\bar{M}}}}-a-b \ln t\right)^{2}}{\left.\sum_{t=1}^{N_{M C}} \overline{\overline{\ln \bar{M}}}-\ln \langle M\rangle(t)\right]^{2}},
$$

with $\overline{\overline{\ln \bar{M}}}=\left(1 / N_{M C}\right) \sum_{t=1}^{N_{M C}} \ln \bar{M}(t)$, and the critical value $K_{c}$ corresponds to $K^{(o p t)}=\arg \max _{K \in\left[K^{(\min )}, K^{(\max )}\right]}\{r\}$. The coefficient $r$ has a very simple explanation: it measures the ratio: (expected variation)/(total variation). The bigger the $r$, the better the linear fit in log scale, and therefore, the better the power law that corresponds to the critical parameter excepted for an order of error $\Delta K$.

Particularly for these simulations, whose main aim is to check the critical parameter, we used only $N_{M C}=300 \mathrm{MC}$ steps but, for the simulations used to estimate the static critical exponents, we used $N_{M C}=1000 \mathrm{MC}$ steps.

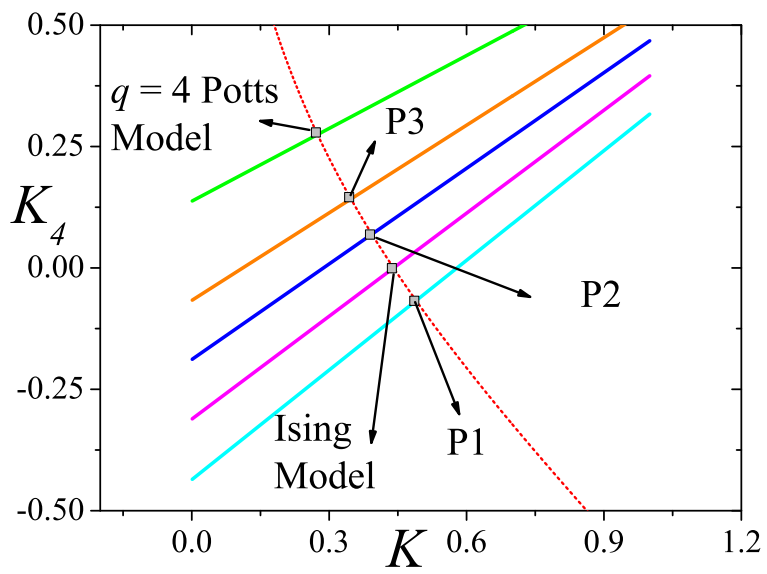

FIG. 1. Critical line described by equation $K_{4}=$ $-\frac{1}{2} \ln [\sinh (2 K)]$. The points correspond to $J=0.4 \quad(\mathrm{P} 1)$, $J=0.5$ (P2), Ising model, $J \simeq 0.6$ (P3), and four-state Potts (FSP) model. The perpendicular lines passing through each point are also presented. 


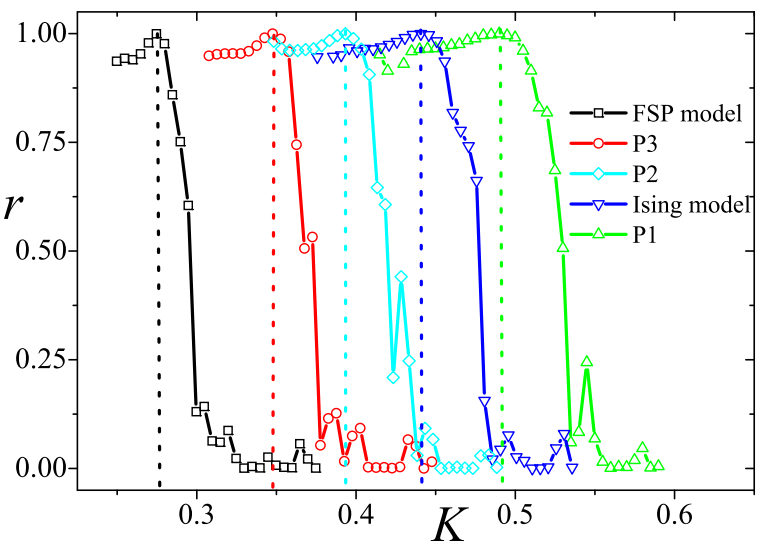

FIG. 2. Coefficient of determination $r$ as function of $K$ walking on the perpendicular line presented in Fig. 1. The maximum occurs at the expected critical points.

In Fig. 2 we can observe that the maximum occurs exactly in the point $\left[K^{(c)}, K_{4}^{(c)}\right]$ as conjectured by Eq. (33) for each point. This figure shows $r$ as function of $K$ walking on the perpendicular line given by Eq. (36). Since we corroborate such conjecture using an optimizer based on MC simulations, we are now prepared to study the critical exponents (dynamic and static ones) for these points.

\section{RESULTS}

In this article we study the short-time critical dynamics of the Ashkin-Teller model [7] by carrying out Monte Carlo simulations in five points (see Table I) along the Baxter line where the model presents nonuniversal behavior.

We estimate the dynamic critical exponents $\theta_{g_{m}}, \theta_{g_{p}}, \theta_{m}, \theta_{p}$, $z_{m}$, and $z_{p}$ for each considered critical point as well as the static critical exponents: $v_{m}, v_{p}, \beta_{m}$, and $\beta_{p}$. We elaborate a more detailed statistical procedure to estimate the static exponents since their sensitivity deserves more attention. Among the points we take into account, we include the critical points of the Ising, and FSP models. The exponents $\theta_{g_{m}}, \theta_{m}$, and $z_{m}$ have already been obtained numerically for the critical points of the two-dimensional Ising model [20,49-52] and the FSP model $[35,37,43,53-55]$. These last two exponents, as well as the exponents $\theta_{p}$ and $z_{p}$ were calculated for some points on the self-dual critical line of the Ashkin-Teller model by Li et al. [56]. In addition, the exponents $z_{m}$ and $z_{p}$ were estimated for some points on the critical line for the Baxter model by Takano [57]. As far as we know, the dynamic critical exponents $\theta_{g_{m}}$, and $\theta_{g_{p}}$ were not found yet for the Ashkin-Teller model. It is important to mention that $\theta_{m}$ and $\theta_{p}$ have not yet been obtained by power-law correlations, as well as the exponents $z_{m}$ and $z_{p}$, which have not yet been studied through the method that mixes initial conditions. Both methods are employed in this paper. On the other hand, for the static exponents, conjectures assert that the ratio $\beta_{m} / v_{m}=1 / 8$ for the entire critical line while $\beta_{p} / v_{p}$ is not constant as $J$ increases. Hence, this fact deserves attention and a detailed study.

In our simulations we use square lattices of linear sizes $L=64,128$, and 256 and the system evolves in contact with a thermal bath in five points on the self-dual critical line of the AT model. Zheng in Ref. [20] has shown that there is no expected differences in exponents for the lattice sizes $L=128$ and 256 within error bars. As will be shown in the following tables, our results assert that, as in Zheng's work, there is no need of performing simulations for lattices greater than $L=256$. Our estimates for each exponent and the corresponding error are obtained from five independent seeds of $N_{\text {run }}$ runs, each one as previously described in Sec. III. However, since the two sublattices of the model $(\sigma$ and $\mu$ ) are symmetrical, the number of effective bins for the magnetization are doubled. In order to measure the slopes of the power laws described above (in double-log scale) we consider the time interval [150,300] for the dynamic exponents. For the static ones, a more detailed statistical tool was prepared taking into consideration averages over different seeds and temporal variations. In this case the maximal number of MC steps was $N_{M C}=10^{3}$.

\section{A. Dynamic critical exponents $\boldsymbol{\theta}_{g_{m}}$ and $\boldsymbol{\theta}_{g_{p}}$}

The first exponents we calculate are the global persistence exponents $\theta_{g_{m}}$ and $\theta_{g_{p}}$ that are achieved when one considers the global persistence probabilities $G_{M}(t)$ and $G_{P}(t)$, Eqs. (20) and (21), which are defined as the probabilities of the order parameters (magnetization and polarization) not changing their signs up to the time $t$, at criticality $(\tau=0)$.

In order to obtain these exponents, one can define the global persistence probability as

$$
G_{M}(t)=1-\sum_{t^{\prime}=1}^{t} \rho_{m}\left(t^{\prime}\right)
$$

and

$$
G_{P}(t)=1-\sum_{t^{\prime}=1}^{t} \rho_{p}\left(t^{\prime}\right),
$$

where $\rho_{m}\left(t^{\prime}\right)$ and $\rho_{p}\left(t^{\prime}\right)$ are the fractions of samples that have changed the sign of their magnetization and polarization, respectively, for the first time at the instant $t^{\prime}$. Here, the simulations are performed for some predefined values of the initial magnetization $m_{0} \ll 1$ and polarization $p_{0} \ll 1$. Hence, a sharp preparation of the initial states is needed to obtain precise values for them. After obtaining the exponents $\theta_{g_{m}}$ and $\theta_{g_{p}}$ for each value of $m_{0}$ and $p_{0}$, respectively, the final values are achieved by performing the limit procedures $m_{0} \rightarrow 0$ and $p_{0} \rightarrow 0$.

In this paper, we consider the following values for $m_{0}$ and $p_{0}: 0.002,0.004,0.006$, and 0.008 . To obtain these values, we first insert randomly, at each site of the sublattices, a spin variable that takes the values \pm 1 . After that, the magnetization of the sublattices and the polarization of the system are measured by using Eq. (8). Then, spin variables are chosen randomly and their signs are changed until we obtain a null value for the magnetizations and polarization. The last procedure is to change the signs of $\delta / 2$ sites of each sublattice at random to obtain the desired initial magnetization $m_{0}$ and polarization $p_{0}$.

In Fig. 3 we show the decay of the global persistence probability of the magnetization (on top) for the five considered points, for $L=256$ and $m_{0}=0.008$. The error bars are smaller than the symbols. In that same figure, at the bottom, we present 

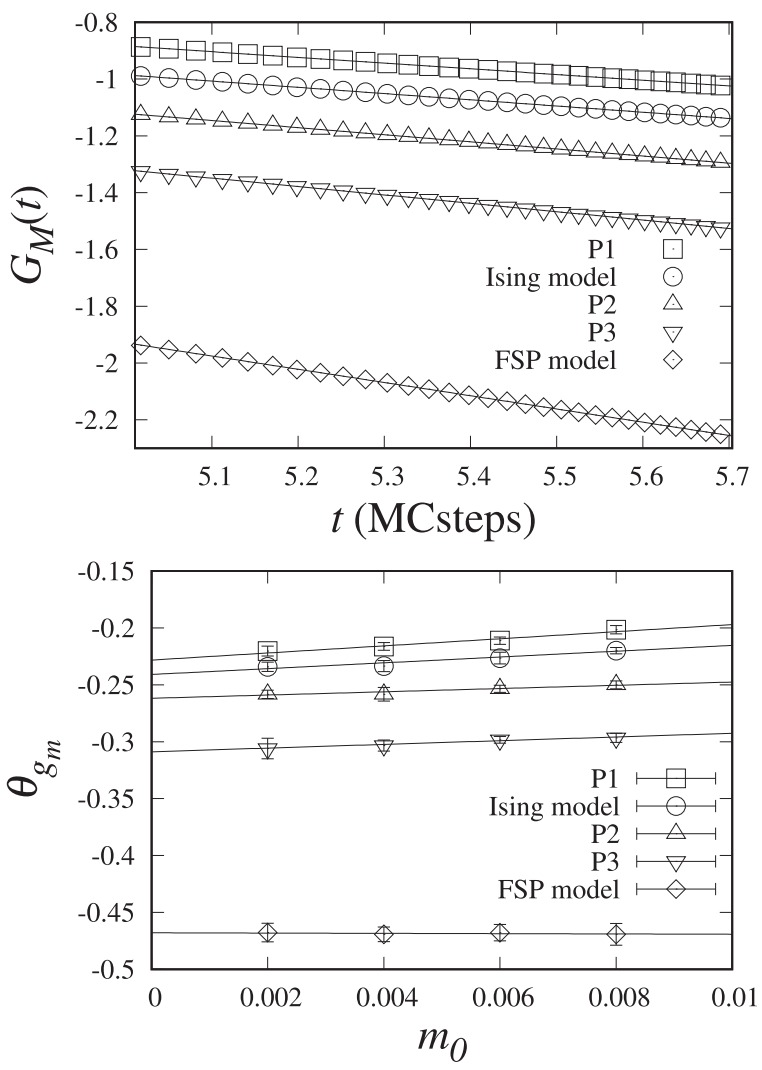

FIG. 3. Global persistence probability of the magnetization for $L=256$. Top: polynomial decay of $G_{M}(t) \times t$ for $m_{0}=0.008$. Bottom: linear fit of the estimates obtained for different values of $m_{0}$.

the plots of $\theta_{g_{m}}$ as function of $m_{0}$, as well as the limit procedure $m_{0} \rightarrow 0$.

Table II presents the results obtained from the limit procedure for the three lattices, $L=64,128$, and 256 .

The results show that the dynamic critical exponent $\theta_{g_{m}}$ grows monotonically with $J$. Moreover, the values obtained for the Ising and FSP models can be compared with results obtained previously and found in literature.

For the Ising critical point, the uncoupled point, our result is in complete agreement with that presented by Schulke et al. [24], $\theta_{g}=0.238(3)$. Our result can also be compared to the value obtained by Majumdar et al. [22] using a finite-size scaling technique. By starting from a random initial configuration and collapsing the data, they found $\theta_{g} z=0.505$ (20). If we consider our estimate for $z_{m}$ (presented

TABLE II. The global persistence exponent $\theta_{g_{m}}$ for the five considered points.

\begin{tabular}{lclc}
\hline \hline CP & $L=64$ & $L=128$ & $L=256$ \\
\hline P1 & $0.2063(26)$ & $0.2211(16)$ & $0.2283(28)$ \\
Ising model & $0.2186(19)$ & $0.2381(40)$ & $0.2409(26)$ \\
P2 & $0.2417(25)$ & $0.2656(7)$ & $0.2618(14)$ \\
P3 & $0.2835(18)$ & $0.3032(20)$ & $0.3089(23)$ \\
FSP model & $0.4678(38)$ & $0.4763(60)$ & $0.4679(13)$ \\
\hline \hline
\end{tabular}

in Sec. V C), $z_{m}=2.156(11)$, one finds $\theta_{g}=0.234(10)$. This result is slightly smaller than the value obtained in this paper but they are in agreement with each other when considering the statistical errors.

For the FSP model, Fernandes et al. [35] obtained $\theta_{g}=$ $0.474(7)$ and Arashiro et al. [55] found $\theta_{g}=0.475$ (5) for the FSP model and $\theta_{g}=0.471(5)$ for the $n=3$ Turban model (this model belongs to the four-state Potts model universality class). Therefore, our estimate is in good agreement with those obtained previously.

Figure 4 shows the global persistence probability in double$\log$ scale for the polarization, for the five points along the self-dual critical line (on top), $L=256$ and $p_{0}=0.008$. The error bars are smaller than the symbols.

The plots of $\theta_{g_{p}}$ as function of $p_{0}$, as well as the limit procedure $p_{0} \rightarrow 0$ are shown at the bottom of this figure and the extrapolated values are presented in Table III.

For the polarization, the global persistence exponent decreases monotonically with $J$ showing, as above, the nonuniversal character of the model. The values of the exponent are higher than for $\theta_{g_{m}}$ but this difference disappears for the four-state Potts critical point whereas in this point $K=K_{4}$ and both $\theta_{g_{m}}$ and $\theta_{g_{p}}$ share the same value.

\section{B. Dynamic critical exponents $\boldsymbol{\theta}_{m}$ and $\boldsymbol{\theta}_{p}$}

As stressed before, we consider two different approaches to estimate the exponents $\theta_{m}$ and $\theta_{p}$. Our first attempt is
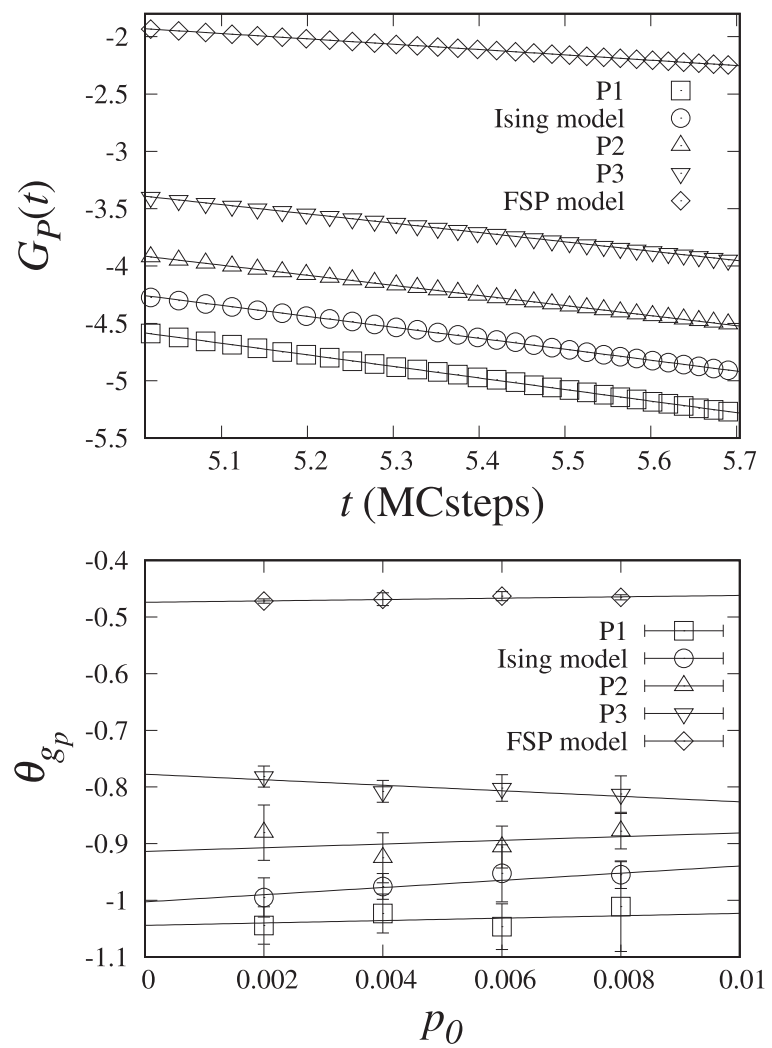

FIG. 4. Global persistence probability of the polarization for $L=256$. Top: polynomial decay of $G_{P}(t) \times t$ for $p_{0}=0.008$. Bottom: linear fit of the estimates obtained for different values of $p_{0}$. 
TABLE III. The global persistence exponent $\theta_{g_{p}}$ for the five considered points.

\begin{tabular}{llll}
\hline \hline CP & \multicolumn{1}{c}{$L=64$} & \multicolumn{1}{c}{$L=128$} & \multicolumn{1}{c}{$L=256$} \\
\hline P1 & $1.143(55)$ & $1.035(12)$ & $1.044(19)$ \\
Ising model & $0.9941(76)$ & $0.9867(57)$ & $1.0027(80)$ \\
P2 & $0.9279(186)$ & $0.9186(114)$ & $0.9137(346)$ \\
P3 & $0.8183(224)$ & $0.7988(94)$ & $0.777(121)$ \\
FSP model & $0.4607(105)$ & $0.4570(106)$ & $0.4741(18)$ \\
\hline \hline
\end{tabular}

related to the time correlation of the magnetization and polarization, Eqs. (12) and (13), respectively. However, the huge fluctuations, even for $3 \times 10^{5}$ samples, prevented us from considering this technique to obtain $\theta_{p}$. In Fig. 5, $Q_{M}(t)$ is plotted in double-log scale for five different points.

Table IV shows our numerical results for each $\theta_{m}$ with the corresponding error, for the three lattice sizes considered in this paper.

The second method consists of calculating the exponents $\theta_{m}$ and $\theta_{p}$ for different values of $m_{0} \ll 0$ and $p_{0} \ll 0$, respectively, by using the Eqs. (14) and (15). Their final values are then obtained by carrying out the limit procedure $m_{0} \rightarrow 0$ and $p_{0} \rightarrow 0$. In order to avoid huge fluctuations of the order parameters, which arise when $m_{0}$ and $p_{0}$ are very close to zero, we consider the following values of $m_{0}$ and $p_{0}: 0.02$, $0.04,0.06$, and 0.08 .

Figure 6 shows the polynomial behavior of $M(t) \times t$ for $m_{0}=0.06$ in double-log scales, for the five different points and $L=256$ (top).

The limit procedures are shown at the bottom of this figure and the extrapolated values can be seen in Table $\mathrm{V}$ for the three lattices, $L=64,128$, and 256 .

Our results displayed in Tables IV and V are in good agreement with each other and show that the exponent $\theta_{m}$ varies continuously with $J$. The estimates also corroborate the available values for the Ising and FSP models. For the former one, our results should be compared with those ones showed by Grassberger [52], $\theta=0.191(3)$, Li et al. [56], $\theta=0.191(2)$, and Okano et al. [51], $\theta=0.191(1)$. For the FSP model, Okano et al. [51] conjectured that the exponent $\theta$ should be negative

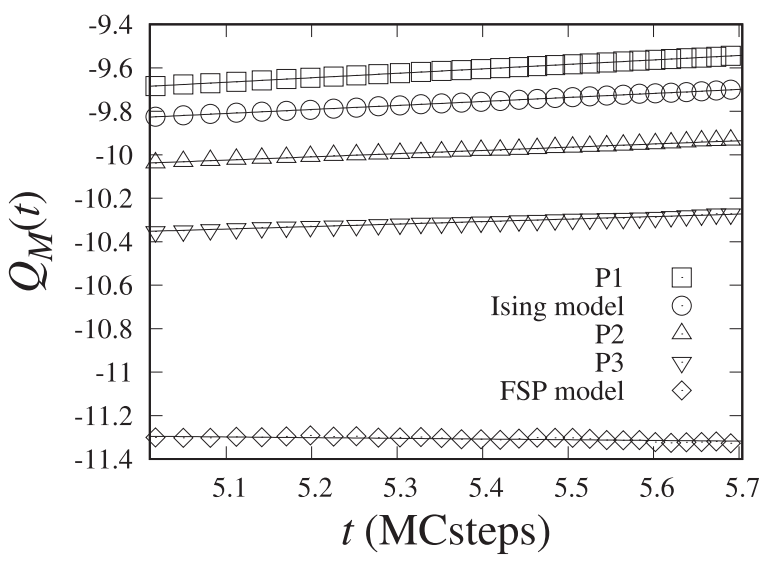

FIG. 5. The time evolution of the time correlation of the magnetization $Q_{M}(t)$.
TABLE IV. The dynamic critical exponent $\theta_{m}$ obtained from the time correlation of the magnetization, $Q_{M}(t)$, for the five coupling constants of the Baxter model at the self-dual critical line of the Ashkin-Teller model.

\begin{tabular}{lrrr}
\hline \hline CP & $L=64$ & $L=128$ & \multicolumn{1}{c}{$L=256$} \\
\hline P1 & $0.207(15)$ & $0.208(13)$ & $0.205(10)$ \\
Ising model & $0.188(20)$ & $0.189(17)$ & $0.188(13)$ \\
P2 & $0.163(10)$ & $0.158(17)$ & $0.162(20)$ \\
P3 & $0.129(19)$ & $0.131(25)$ & $0.121(17)$ \\
FSP model & $-0.087(85)$ & $-0.071(77)$ & $-0.031(51)$ \\
\hline \hline
\end{tabular}

and close to zero and the results for this model [54,55] as well as for the Ising model with three-spin interactions [58] validate this assertion. Besides the four-state Potts model, it has been shown in some papers that there are models in which the exponent $\theta$ can also have a negative value, for instance, the tricritical Ising model [59], Blume-Capel model [38], metamagnetic model [39], and Baxter-Wu model [43,60].

In order to obtain the exponent $\theta_{p}$, we consider the same initial conditions, i.e., $p_{0}=0.02,0.04,0.06$, and 0.08 . Figure 7 displays the behavior of $P(t) \times t$, Eq. (15), in double-log scale for $p_{0}=0.08$ and $L=256$ for the five critical points considered.

The extrapolated values, obtained from the limit procedure $p_{0} \rightarrow 0$, are presented in Table VI for the three lattices, $L=64,128$, and 256 .
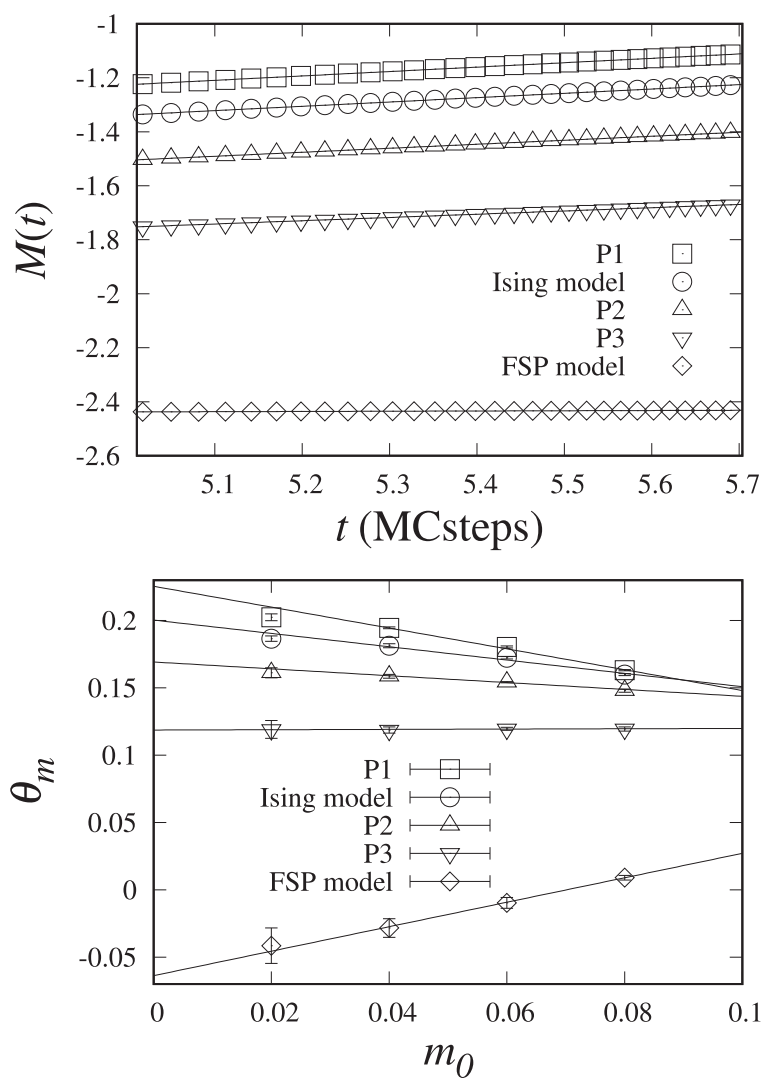

FIG. 6. The plot of $M(t) \times t$, Eq. (14), for the initial magnetization $m_{0}=0.08$ and $L=256$. Top: polynomial behavior of $M(t) \times t$. Bottom: linear fit of the estimates obtained for different values of $m_{0}$. 
TABLE V. The dynamic critical exponent $\theta_{m}$ for the five considered points.

\begin{tabular}{lccr}
\hline \hline CP & $L=64$ & $L=128$ & \multicolumn{1}{c}{$L=256$} \\
\hline P1 & $0.2171(23)$ & $0.2198(35)$ & $0.2203(45)$ \\
Ising model & $0.1915(16)$ & $0.1999(20)$ & $0.1951(26)$ \\
P2 & $0.1681(64)$ & $0.1646(33)$ & $0.1666(14)$ \\
P3 & $0.1172(19)$ & $0.1196(9)$ & $0.1176(10)$ \\
FSP model & $-0.0580(21)$ & $-0.0707(59)$ & $-0.0611(42)$ \\
\hline \hline
\end{tabular}

The results show that the exponent $\theta_{p}$ decreases monotonically with respect to $J$. They are completely different from those obtained by Li et al. [56], but for the four-state Potts critical point ( $y=1$ in that paper). They showed that the polarization is negative for all considered points.

\section{Dynamic critical exponents $z_{m}$ and $z_{p}$}

Finally, the dynamic critical exponents $z_{m}$ and $z_{p}$ are obtained by combining results from samples submitted from different initial conditions (ordered state for the order parameter and disordered one for the second moment of the order parameter), Eqs. (23) and (24), where the dimension of the system is $d=2$. This technique has proven to be very efficient in estimating the exponent $z$ for a large number of models [35,37,38,43,44,55].
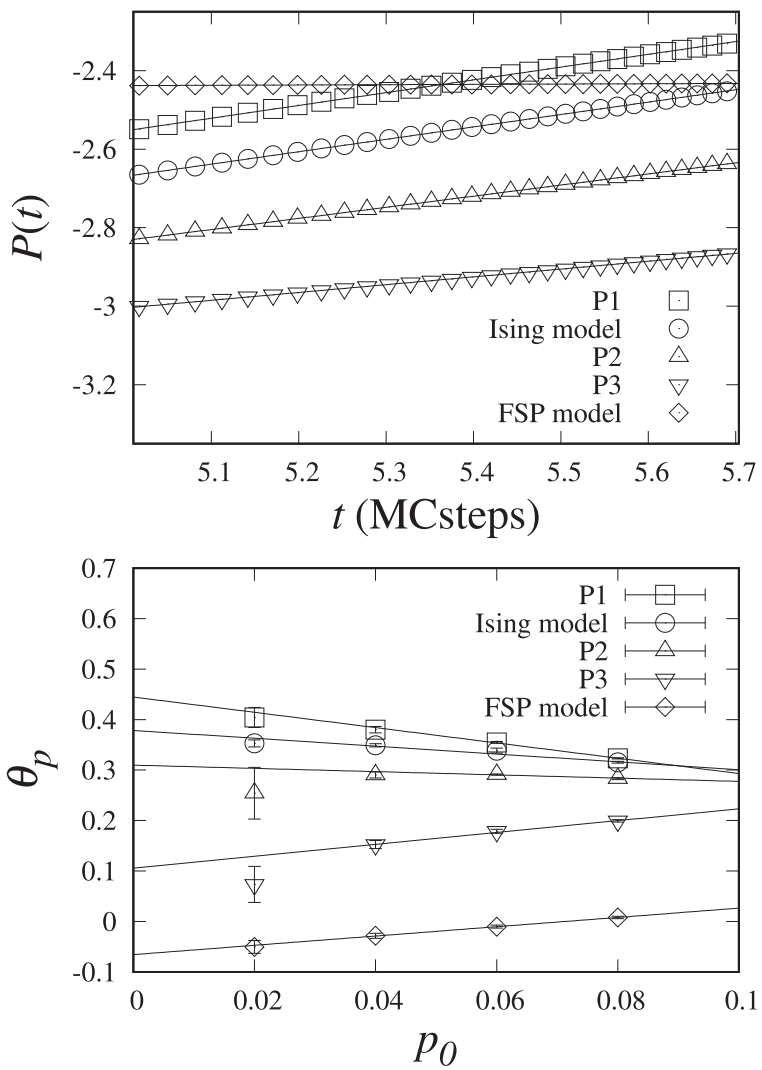

FIG. 7. The plot of $P(t) \times t$, Eq. (15), for the initial polarization $p_{0}=0.08$ and $L=256$. Top: polynomial behavior of $P(t) \times t$. Bottom: linear fitting of the estimates obtained for different values of $p_{0}$.
TABLE VI. The dynamic critical exponent $\theta_{p}$ for the five considered points.

\begin{tabular}{lccc}
\hline \hline CP & \multicolumn{1}{c}{$L=64$} & \multicolumn{1}{c}{$L=128$} & \multicolumn{1}{c}{$L=256$} \\
\hline P1 & $0.4466(320)$ & $0.4364(16)$ & $0.4301(7)$ \\
Ising model & $0.4317(39)$ & $0.3857(94)$ & $0.3638(58)$ \\
P2 & $0.2797(361)$ & $0.3017(125)$ & $0.2860(115)$ \\
P3 & $0.1403(372)$ & $0.0937(210)$ & $0.1106(59)$ \\
FSP model & $-0.0611(115)$ & $-0.0597(44)$ & $-0.0645(1)$ \\
\hline \hline
\end{tabular}

The time evolution of $F_{2_{M}}$, obtained from Eq. (23), is shown in Fig. 8 in double-log scale for the five considered points and $L=256$. The error bars are smaller than the symbols.

The mean values of $z_{m}$ and the corresponding errors are given in Table VII for $L=64,128$, and 256.

In the decoupling point, $J=K=0.5 \ln (1+\sqrt{2})$, the exponent $z_{m}$ is in complete agreement with those obtained for the two-dimensional Ising model [50,51]. However, our estimate of $z_{m}$ for the FSP model, is larger, but very close to the values recently obtained for that model, $z=2.290$ (3) [37] and $z=2.294(3)$ [35], for the Baxter-Wu model [43], $z=$ 2.294(6), and for the $n=3$ Turban model [55], $z=2.292(4)$, both belonging to the same universality class of the FSP model.

In Fig. 9 we show the time dependence of $F_{2_{P}}(t)$ in double$\log$ scales for the five considered points and $L=256$. The error bars, obtained from five independent runs, are smaller then the symbols. The linear fits of these curves, as well as of those ones with $L=64$ and $L=128$, lead to the values presented in Table VIII.

By taking into account the statistical errors, the results shown in Tables VII and VIII ensure that the exponents $z_{m}$ and $z_{p}$ are varying with respect to $J$. In this case, only the exponent for the FSP model is different from the others. Besides, for this critical point, the exponents $z_{m}$ and $z_{p}$ share the same values (within the error bars). On the contrary, the exponent $z_{p}$ is greater than $z_{m}$ for the other points, in contrast with the results shown in Ref. [56] for the Ashkin-Teller model, where there is no distinction between the two critical indexes.

\section{Static critical exponents}

Here we finally calculate the static critical exponents of the AT model. By using the exponents $z_{m}$ and $z_{p}$ obtained

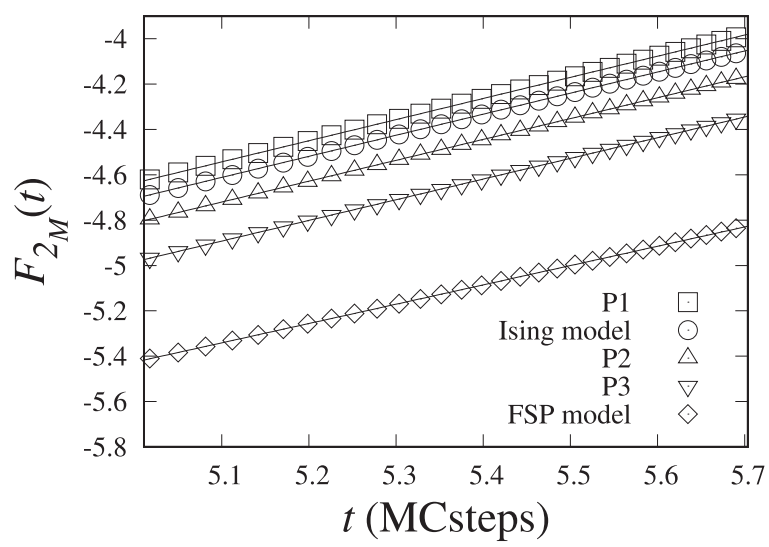

FIG. 8. Time evolution of $F_{2_{M}}(t)$ for the five critical points and $L=256$. 
TABLE VII. The dynamic critical exponent $z_{m}$ for the five considered points.

\begin{tabular}{llll}
\hline \hline CP & $L=64$ & $L=128$ & $L=256$ \\
\hline P1 & $2.113(18)$ & $2.139(16)$ & $2.147(12)$ \\
Ising model & $2.129(12)$ & $2.156(11)$ & $2.156(11)$ \\
P2 & $2.154(9)$ & $2.168(13)$ & $2.172(13)$ \\
P3 & $2.175(5)$ & $2.198(14)$ & $2.194(17)$ \\
FSP model & $2.318(11)$ & $2.342(21)$ & $2.346(21)$ \\
\hline \hline
\end{tabular}

in the previous section, we calculate the exponent $v$ for the magnetization and polarization, respectively. Differently from what occurs with the dynamic exponents, the computing of static exponents deserves a more detailed analysis of uncertainties and of final estimates. In this analysis we consider both the external and internal averages.

In this paper, the static exponents were calculated by using $N_{\text {run }}=4000$ runs in order to compute the averaged time series in the situation that the system starts from $m_{0}=1$ (or $p_{0}=1$ ). First, the error bars are obtained with $N_{b}=5$ different bins (for polarization). For the case of magnetization we have $N_{b}=10$ different bins since the lattices are doubled. Here it is important to differentiate bin from seed. We always used five seeds, but due to duplicity of lattices in the AT model, and considering the isotropic case $\left(K_{1}=K_{2}=K\right)$ the number of bins is equal to the double of seeds for the magnetization case.

We numerically compute the derivative through Eqs. (29) and (30), which leads to:

$$
D(t)=\frac{1}{2 \delta} \ln \frac{\bar{O}\left[K_{4}\left(T_{c}+\varepsilon\right), K\left(T_{c}+\varepsilon\right), t\right]}{\bar{O}\left[K_{4}\left(T_{c}-\varepsilon\right), K\left(T_{c}-\varepsilon\right), t\right]},
$$

where $\bar{O}\left[K_{4}(T), K(T), t\right]$ denotes the averaged magnetization and polarization calculated in values above and below the critical temperatures. If $K\left(T_{c}\right)=K_{c}=\frac{1}{T_{c}}$, it is interesting to observe that a perturbation on the critical temperature, $T_{c} \pm \varepsilon$, produces $K\left(T_{c} \pm \varepsilon\right)=1 /\left(T_{c} \pm \varepsilon\right)=\frac{1 / T_{c}}{\left(1 \pm \varepsilon / T_{c}\right)}=K_{c} /(1 \pm \delta)$, where $\delta=\varepsilon / T_{c}$. This means that when we divide $K_{c}$ by $(1 \pm$ $\delta)$, the critical temperature is perturbed by a value $\pm \varepsilon= \pm \delta T_{c}$. Similarly, $K_{4}\left(T_{c} \pm \varepsilon\right)=K_{4}^{c} /(1 \pm \delta)$.

Figure 10 shows the time evolution of $D(t)$ for magnetization and polarization, calculated by Eq. (40). It is important to

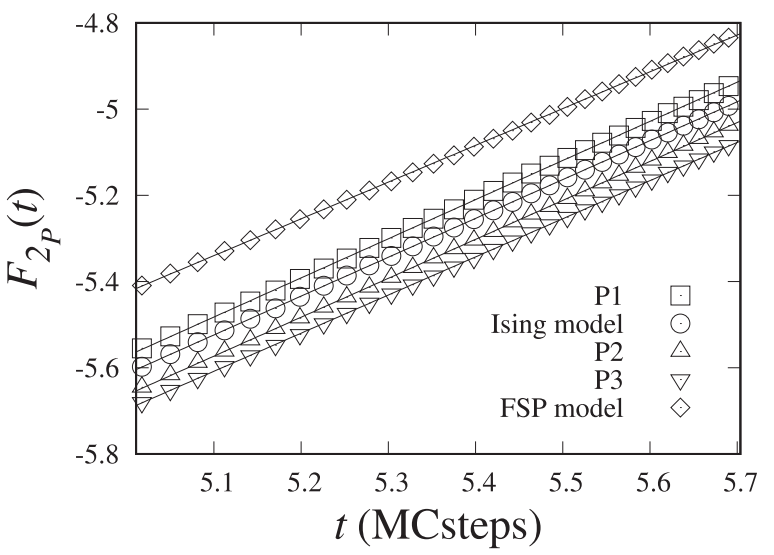

FIG. 9. Time evolution of $F_{2_{P}}(t)$ for the five critical points.
TABLE VIII. The dynamic critical exponent $z_{p}$ for the five considered points.

\begin{tabular}{llll}
\hline \hline CP & $L=64$ & $L=128$ & $L=256$ \\
\hline P1 & $1.889(19)$ & $2.236(16)$ & $2.201(8)$ \\
Ising model & $1.932(25)$ & $2.225(21)$ & $2.220(16)$ \\
P2 & $2.004(25)$ & $2.232(17)$ & $2.212(26)$ \\
P3 & $2.092(10)$ & $2.253(19)$ & $2.258(17)$ \\
FSP model & $2.210(12)$ & $2.341(12)$ & $2.338(38)$ \\
\hline \hline
\end{tabular}

notice that the best $\delta$ ( $\left.\delta_{\text {best }}\right)$ for each critical point can change and we study the best lag $\delta_{\text {best }}$ suitable for each point. For the magnetization, the error bars are obtained by using an average over different $N_{b}^{2}=100$ points while for the polarization $N_{b}^{2}=25$ points since we cross the $N_{b}$ time series simulated above critical parameter: $\bar{O}\left(T_{c}+\varepsilon, t\right)$, on the perpendicular line as previously described, with $N_{b}$ time series simulated below critical parameter $\bar{O}\left(T_{c}-\varepsilon, t\right)$. We can clearly observe a power-law behavior (log-log plot) for all points studied.

So, in order to compute the static exponents, we observe that the exponent $\phi=1 /(v z)$ has an important variation on the different time lags considered, and therefore, such a variation must be considered in the final estimates of the exponent $v$. In this analysis we index by $k$ the time lag $\left[t_{i}^{(k)}, t_{f}^{(k)}\right]$, where $k=1, \ldots, n$. It was built considering that the minimum size of the interval is $\Delta=100 \mathrm{MC}$ steps. Moreover, the minimum $t_{i}$ adopted is $50 \mathrm{MC}$ steps, while the maximum $t_{f}$ is $1000 \mathrm{MC}$ steps.
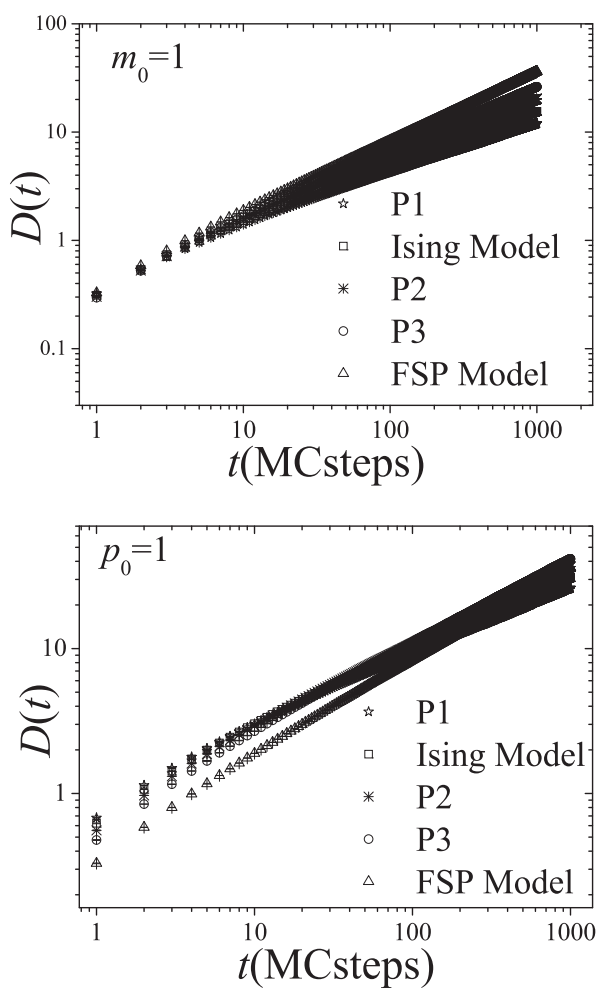

FIG. 10. Time evolution of $D(t)$ for the five coupling constants. Top: magnetization. Bottom: polarization. 

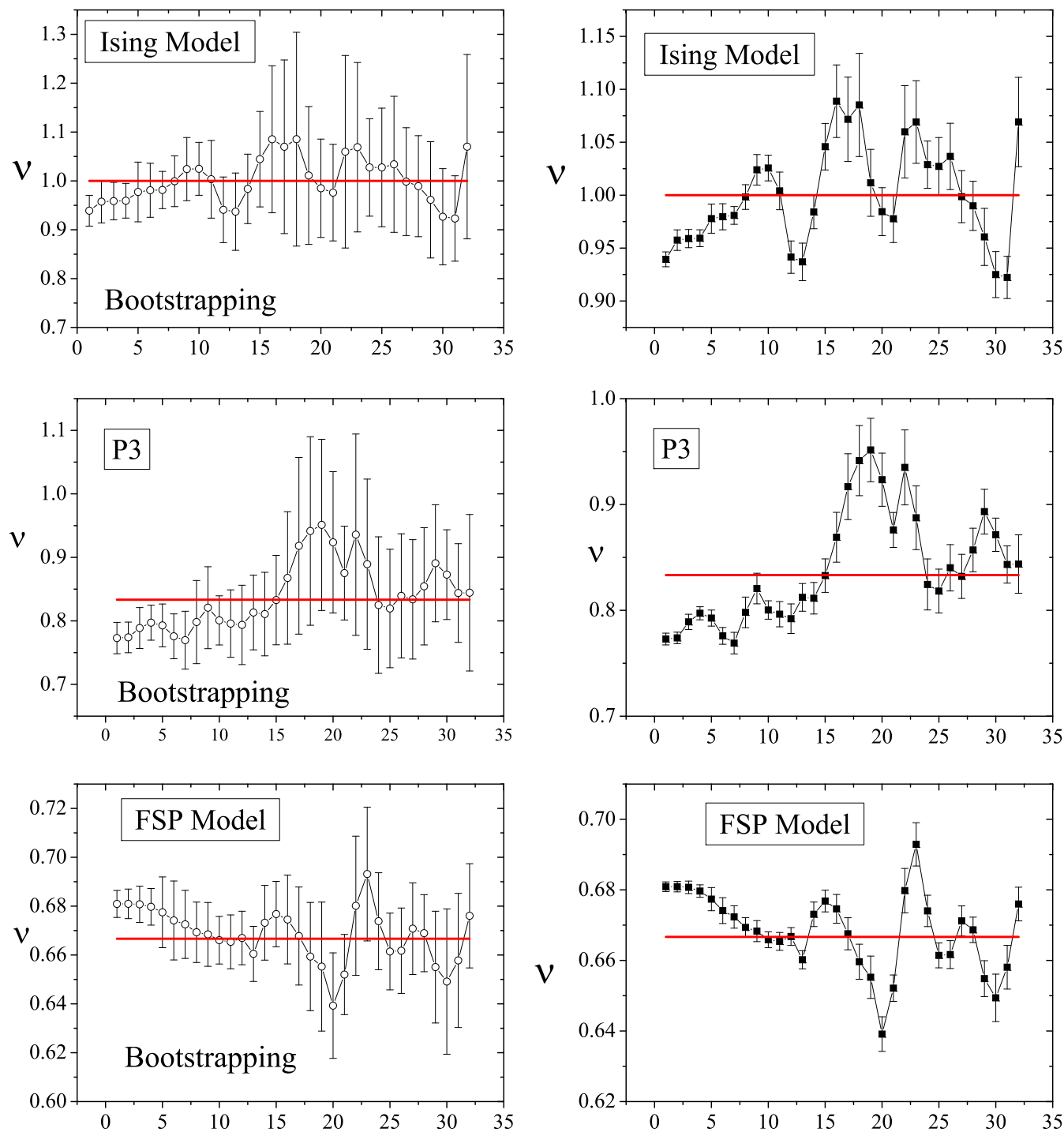

FIG. 11. Estimates of the exponents $v_{m}$ for the different time lags. The left-side plots show the exponents obtained by bootstrapping method by adopting $N_{\text {sample }}=10^{3}$ while the right-side ones were obtained with a simple crossing of $N_{b}=10$ seeds (100 points).

We prepared an algorithm that considers the same number of points per interval, which allows to perform linear fits under the same conditions for all different intervals considered in the analysis. The appropriate number of points per interval in this paper was $n_{p}=25$, and the spacing was adjusted to satisfy such restriction.

Let us denote here $\bar{O}_{l}\left(T_{c}+\varepsilon, t\right)$, the order parameter averaged over $N_{\text {run }}$ different runs corresponding to the $l$ th bin calculated in $T_{c}+\varepsilon$ and $\bar{O}_{m}\left(T_{c}-\varepsilon, t\right)$ corresponding to the $m$ th bin that was calculated in $T_{c}-\varepsilon$. Denoting $\phi_{k}^{(l, m)}$ the exponent $1 / \mathrm{vz}$, calculated using the two bins $l$ and $m$ previously reported for the time lag $k$ after a fitting of the power law $\frac{1}{2 \delta} \ln \frac{\bar{O}_{l}\left(T_{c}+\varepsilon, t\right)}{\bar{O}_{m}\left(T_{c}-\varepsilon, t\right)} \propto t^{\phi(, m)}$, our final estimate of the exponent $\phi_{k}$ in this time lag is

$$
\phi_{k}=\frac{1}{N_{b}^{2}} \sum_{l, m=1}^{N_{b}} \phi_{k}^{(l, m)}
$$

which is an average over the bins. In this case we have an uncertainty given by

$$
\sigma_{k}^{2}=\frac{1}{N_{b}^{2}\left(N_{b}^{2}-1\right)} \sum_{l, m=1}^{N_{b}}\left(\phi_{k}^{(l, m)}-\phi_{k}\right)^{2} .
$$

Finally, we have the final estimate $\phi=\frac{1}{n} \sum_{k=1}^{n} \phi_{k}$, which is an average over the time lags and which leads to a final uncertainty:

$$
\begin{aligned}
\sigma_{\phi}^{2} & =\frac{1}{n(n-1)} \sum_{k=1}^{n}\left(\phi_{k}-\phi\right)^{2}+\frac{1}{n^{2}} \sum_{k=1}^{n} \sigma_{k}^{2} \\
& =\sigma_{\mathrm{ext}}^{2}+\sigma_{\mathrm{int}}^{2},
\end{aligned}
$$

where the first term of the right-hand side corresponds to external uncertainty (variation over the time lags). This term corresponds to a temporal variation of the exponent, since the exponent estimated for each lag changes along the time evolution. A second source of errors corresponds to 
TABLE IX. The static critical exponents $v$ for the five considered points, for magnetization $(m)$ and polarization $(p)$. All estimates were obtained for the largest lattice used in this work: $L=256$. Here $\delta_{\text {best }}$ is the best lag used in derivatives.

\begin{tabular}{|c|c|c|c|c|c|c|c|c|c|c|}
\hline $\mathrm{CP}$ & $v_{m}$ & $v_{m}^{(\text {boot })}$ & $v_{m}^{(\min )}$ & $v_{m}^{(\max )}$ & $v_{p}$ & $v_{p}^{(\text {boot })}$ & $v_{p}^{(\min )}$ & $v_{p}^{(\max )}$ & $v$ [Eq. (34)] & $\delta_{\text {best }}$ \\
\hline $\mathrm{P} 1$ & $1.060(12)$ & $1.060(24)$ & $1.009(28)$ & $1.117(30)$ & $1.021(12)$ & $1.021(16)$ & $0.997(48)$ & $1.066(52)$ & 1.08900 & 0.005 \\
\hline Ising model & $1.001(19)$ & $1.000(42)$ & $0.922(38)$ & $1.089(68)$ & $0.974(12)$ & $0.974(28)$ & $0.911(14)$ & $1.020(88)$ & 1.00000 & 0.002 \\
\hline P2 & $0.893(14)$ & $0.893(24)$ & $0.844(19)$ & $0.941(46)$ & $0.862(11)$ & $0.862(18)$ & $0.820(20)$ & $0.912(42)$ & 0.91500 & 0.002 \\
\hline P3 & $0.839(19)$ & $0.839(36)$ & $0.773(11)$ & $0.951(58)$ & $0.807(20)$ & $0.807(28)$ & $0.749(15)$ & $0.904(86)$ & $0.8333 \overline{3}$ & 0.001 \\
\hline FSP model & $0.6682(41)$ & $0.6684(74)$ & $0.6391(98)$ & $0.693(12)$ & $0.6679(58)$ & $0.6680(82)$ & $0.637(11)$ & $0.687(17)$ & $0.6666 \overline{6}$ & 0.002 \\
\hline
\end{tabular}

the internal component of uncertainty, i.e., the variation over the pairs of the different seeds used for each time lag. So by a final estimate of uncertainty we must compose these two sources.

For the more skeptical, we also elaborate a bootstrapping version of this analysis. The idea of bootstrapping is to supply error bars, which would be more consistent by using a larger number of seeds and so necessarily demanding higher computational times.

In this case we choose two sets of five seeds (which should be repeated as prescribed by the bootstrapping method) and compose two new time series by averaging them. The first one corresponding to the parameter $T_{c}+\varepsilon$ and the other one corresponding to the parameter $T_{c}-\varepsilon$ yields an exponent. We can repeat this procedure $N_{\text {sample times instead of taking }}$ the $N_{b}^{2}$ possible pairs as the previously described method. So we can replace the Eqs. (41) and (42) by their corresponding bootstrap equations : $\phi_{k}=\frac{1}{N \text { sample }} \sum_{i_{s}=1}^{N \text { sample }} \phi_{k}^{\left(i_{s}\right)}$ and $\sigma_{k}^{2}=\frac{1}{\left(N_{\text {sample }}-1\right)} \sum_{i_{s}=1}^{N_{\text {sample }}}\left(\phi_{k}^{\left(i_{s}\right)}-\phi_{k}\right)^{2}$, respectively, where $\phi_{k}^{\left(i_{s}\right)}$ denotes the exponent calculated for the $i_{s}$ th element of the sample for the $k$ th time lag. Equation (43) remains the same. Given the exponent $z \pm \sigma_{z}$ previously calculated, the final estimate of $v$ is obtained as $v=(z \phi)^{-1}$, and the uncertainty is obtained by $\sigma_{v}=v \sqrt{\left(\frac{\sigma_{z}}{z}\right)^{2}+\left(\frac{\sigma_{\phi}}{\phi}\right)^{2}}$.

First, in order to observe the variation of the exponent $v$ over the different time lags we prepare a plot to show the variation of $v$ and its respective error bars for the different parts (time lags) of the power law (Fig. 11). The $x$ axis denotes a number that indexes one specific time lag. It is important to mention that it is a simple ordering, whereas we do not know which time lag corresponds to the specific exponent since we are interested only in observing the fluctuations of this exponent. In this figure, we show the exponents of the magnetization for the points corresponding to the Ising model, P3, and FSP model where the right-hand side corresponds to estimates obtained by using bootstrapping and the left-hand side the regular method (both previously described).

This plot shows that the estimates can be deeply changed along the power law but the theoretical prediction is corroborated. The bootstrapping method produces higher error bars as expected. So, taking into account the different source variations, we obtain estimates to the exponent $v$ for the different points studied in this paper. In Table IX we present our final estimates of this exponent. The term (boot) refers to exponents obtained using bootstraping. The terms max and min mean the largest and smallest values found in our analysis. The conjectured values are shown in the last column and denoted by an asterisk, and are expected to share the same value for both magnetization $(m)$ and polarization $(p)$. We can observe a good agreement between the conjectured values and our estimates.

It is important to mention that we have obtained such exponents by MC simulations, and even by equilibrium MC simulations. The agreement between the exponents $v$ for the polarization and magnetization was only a conjecture.

By following the same process, we analyze the decay of magnetization and polarization described by Eqs. (25) and (26). The time evolving of these amounts are shown in Fig. 12.

Here we proceed exactly as before to calculate $v$. We analyze the external (over different time lags) and internal (over different bins) variations to estimate the exponent $\mu=$ $\beta /(\nu z)$. After a final estimate of $\mu$ and with the previous estimates of $v$ and $z$, we obtain an uncertainty for $\beta$ : $\sigma_{\beta}^{2}=$ $\beta^{2}\left[\left(\frac{\sigma_{\mu}}{\mu}\right)^{2}+\left(\frac{\sigma_{v}}{v}\right)^{2}+\left(\frac{\sigma_{z}}{z}\right)^{2}\right]$. We present our estimates of $\beta$ in Table $\mathrm{X}$ as we did for $v$ in Table IX.
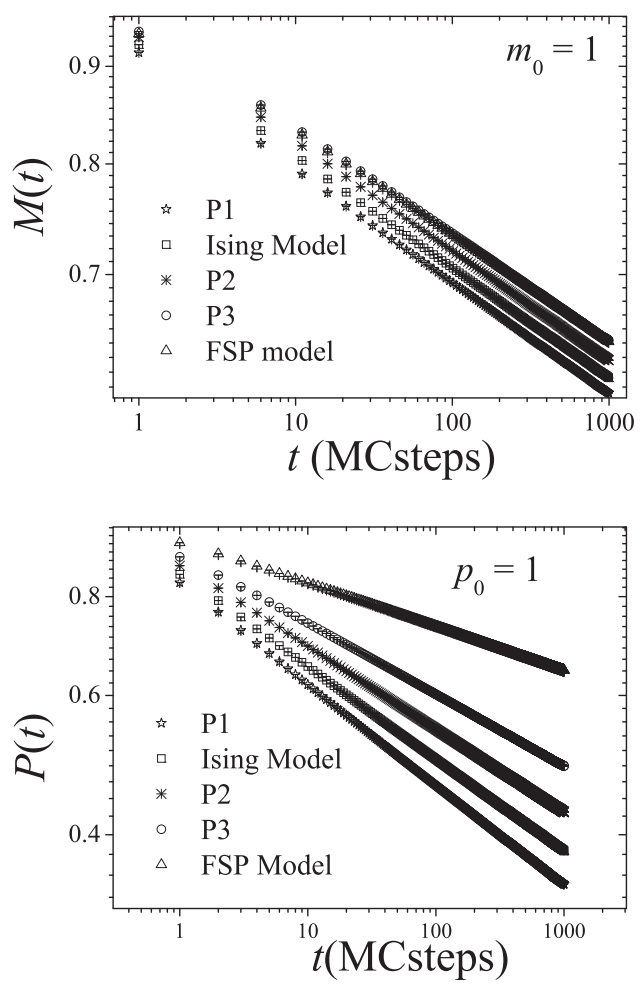

FIG. 12. Time evolving of $\bar{M}(t)$ (top) and $\bar{P}(t)$ (bottom), for $m_{0}=1$ (or $p_{0}=1$ ) for the five coupling constants. 
TABLE X. The static critical exponents $\beta$ for the five considered points, for magnetization $(m)$ and polarization $(p)$. All estimates were obtained for the largest lattice used in this work: $L=256$. Differently from $v$ the conjectured values $\beta^{*}$ for the magnetization and polarization are different.

\begin{tabular}{|c|c|c|c|c|c|c|c|c|c|c|}
\hline $\mathrm{CP}$ & $\beta_{m}$ & $\beta_{m}^{\text {(boot) }}$ & $\beta_{m}^{(\min )}$ & $\beta_{m}^{(\max )}$ & $\beta_{p}$ & $\beta_{p}^{\text {(boot) }}$ & $\beta_{p}^{(\min )}$ & $\beta_{p}^{(\max )}$ & $\beta_{m}^{*}[$ Eq. (34)] & $\beta_{p}^{*}[$ Eq. (34)] \\
\hline $\mathrm{P} 1$ & $0.1325(19)$ & $0.1326(24)$ & $0.1262(24)$ & $0.1409(49)$ & $0.2820(43)$ & $0.2820(43)$ & $0.2711(31)$ & $0.2990(12)$ & 0.1360930 & 0.2943721 \\
\hline Ising model & $0.1246(15)$ & $0.1241(20)$ & $0.1192(58)$ & $0.1310(30)$ & $0.2504(31)$ & $0.2504(31)$ & $0.2404(56)$ & $0.2660(57)$ & 0.1250000 & 0.2500000 \\
\hline $\mathrm{P} 2$ & $0.1114(15)$ & $0.1100(18)$ & $0.1039(58)$ & $0.1173(40)$ & $0.1996(30)$ & $0.1996(28)$ & $0.1927(43)$ & $0.2074(75)$ & 0.1143817 & 0.2075268 \\
\hline P3 & $0.1045(11)$ & $0.1046(15)$ & $0.1024(15)$ & $0.1092(33)$ & $0.1663(22)$ & $0.1663(21)$ & $0.1566(55)$ & $0.1766(50)$ & 0.1041667 & 0.1666667 \\
\hline FSP model & $0.08554(81)$ & $0.0853(11)$ & $0.0825(13)$ & $0.0895(17)$ & $0.0856(10)$ & $0.0858(11)$ & $0.0814(43)$ & $0.0900(50)$ & 0.08333334 & 0.0833334 \\
\hline
\end{tabular}

Differently from what happens for $v$ (Table IX), the conjectured values of $\beta$, for magnetization and polarization, are different and our simulations corroborate both values. It is important to notice that the exponents are the same for the FSP point. In order to test the consistence of the estimates for $\beta$ and $\nu$, we can compare $\beta / \nu$ with conjectured values (see, for example, Ref. [61]). It is also important to stress that $\beta$ and $v$ may not be the same used in other papers and a comparison must be done with some care.

Figure 13 shows the ratio $\beta / \nu$ for the different points. We can check that $\beta / \nu$ remains the same for all points in the case of magnetization (top), while we have a decrease of this ratio when $J$ increases for the polarization. In both situations, an agreement with the conjectured values can be observed. The blue curve was obtained using splines with the five conjectured
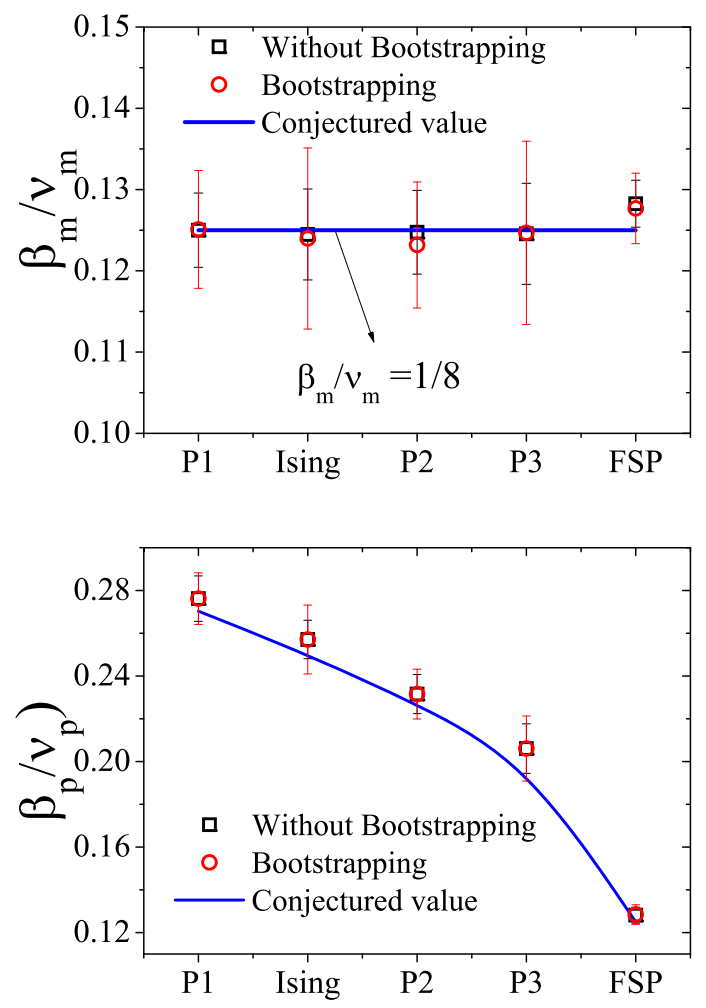

FIG. 13. The ratio $\beta / v$ calculate for magnetization (top) and polarization (bottom). Our values present an excellent agreement with the conjecture. points obtained from literature. So we can check that all exponents and, moreover, the conjectures are in agreement with our time-dependent Monte Carlo simulations, i.e., the exponents can be obtained even out of equilibrium extending even more the applicability of this wide and successful approach.

\section{CONCLUSION}

In this paper, we studied the nonequilibrium critical behavior of the Ashkin-Teller model by performing Monte Carlo simulations far from equilibrium. First, we checked the critical parameters of the model by using a refinement method proposed by the authors of this paper in previous works. After that, the dynamic critical exponents $\theta_{g}, \theta$, and $z$ were obtained for the two order parameters of the model: the magnetization and polarization. The simulations were carried out at five different points on the self-dual critical line including the Ising and four-state Potts critical points. The exponents obtained at those points, for the magnetization, are in good agreement when compared with previous estimates except by the exponent $z_{m}$ for the four-state Potts critical point that is slightly larger than that found in literature.

We have also obtained the static exponents. After a careful procedure to obtain the exponents our results presented a good agreement with conjectured results from literature. The ratio $\beta / v$ decreases when $J$ increases for the polarization but remains the same (according to the error bars) for the magnetization.

In this work, we showed again the wide applicability of the theory of short-time dynamics to describe critical phenomena retrieving equilibrium parameters in simulations out of equilibrium as well as predicting nonequilibrium critical indexes. As an important additional contribution, we also proposed a statistical approach to estimate exponents in time-dependent MC simulations by composing fluctuations from intratime and intertime lags to produce suitable error bars.

\section{ACKNOWLEDGMENTS}

This research work was in part supported financially by CNPq (National Council for Scientific and Technological Development). R.d.S. would like to thank Prof. L. G. Brunnet (IF-UFRGS) for kindly providing the computational resources from Clustered Computing (cluster-slurm.if.ufrgs.br). 
[1] R. J. Baxter, Phys. Rev. Lett. 26, 832 (1971).

[2] B. Widom, J. Chem. Phys. 43, 3898 (1965).

[3] M. E. Fisher, Phys. Rev. Lett. 16, 11 (1966).

[4] L. P. Kadanoff, W. Gotze, D. Hamblen, R. Hecht, E. A. S. Lewis, V. V. Palciaus, M. Rayl, J. Swift, D. Aspnes, and J. Kane, Rev. Mod. Phys. 39, 395 (1967).

[5] L. P. Kadanoff and F. J. Wegner, Phys. Rev. B 4, 3989 (1971).

[6] F. Y. Wu, Phys. Rev. B 4, 2312 (1971).

[7] R. J. Baxter, Exactly Solvable Models in Statistical Physics (Academic Press, London, 1972).

[8] M. N. Barber, J. Phys. A 12, 679 (1979).

[9] J. Ashkin and E. Teller, Phys. Rev. 64, 178 (1943).

[10] C. Fan, Phys. Rev. B 6, 902 (1972).

[11] F. Wegner, J. Phys. C 5, L131 (1972).

[12] L. P. Kadanoff, Phys. Rev. Lett. 39, 903 (1977).

[13] L. P. Kadanoff and A. C. Brown, Ann. Phys. 121, 318 (1979)

[14] J. R. Drugowich de Felicio and R. Koberle, Phys. Rev. B 25, 511 (1982).

[15] R. H. Swendsen and J. S. Wang, Phys. Rev. Lett. 58, 86 (1987).

[16] S. N. Evangelou and E. N. Economou, Phys. Rev. Lett. 68, 361 (1992).

[17] H. K. Janssen, B. Schaub, and B. Schmittmann, Z. Phys. B: Condens. Matter 73, 539 (1989).

[18] D. A. Huse, Phys. Rev. B 40, 304 (1989).

[19] Z. B. Li, L. Schulke, and B. Zheng, Phys. Rev. Lett. 74, 3396 (1995).

[20] B. Zheng, Int. J. Mod. Phys. B 12, 1419 (1998).

[21] T. Tomé and M. J. de Oliveira, Phys. Rev. E 58, 4242 (1998).

[22] S. N. Majumdar, A. J. Bray, S. J. Cornell, and C. Sire, Phys. Rev. Lett. 77, 3704 (1996).

[23] S. N. Majumdar and A. J. Bray, Phys. Rev. Lett. 91, 030602 (2003).

[24] L. Schulke and B. Zheng, Phys. Lett. A 233, 93 (1997).

[25] K. Oerding, S. J. Cornell, and A. J. Bray, Phys. Rev. E 56, R25 (1997).

[26] R. da Silva, N. A. Alves, and J. R. Drugowich de Felício, Phys. Rev. E 67, 057102 (2003).

[27] R. da Silva and N. Alves, Phys. A 350, 263 (2005).

[28] F. Ren and B. Zheng, Phys. Lett. A 313, 312 (2003).

[29] E. V. Albano and M. A. Muñoz, Phys. Rev. E 63, 031104 (2001).

[30] M. Saharay and P. Sen, Phys. A 318, 243 (2003).

[31] H. Hinrichsen and H. M. Koduvely, Eur. Phys. J. B 5, 257 (1998).

[32] P. Sen and S. Dasgupta, J. Phys. A: Math. Gen. 37, 11949 (2004).

[33] B. Zheng, Mod. Phys. Lett. B 16, 775 (2002).

[34] H. A. Fernandes and J. R. Drugowich de Felício, Phys. Rev. E 73, 057101 (2006).

[35] H. A. Fernandes, E. Arashiro, J. R. Drugowich de Felício, and A. A. Caparica, Physica A 366, 255 (2006).
[36] H. A. Fernandes, Roberto da Silva, and J. R. Drugowich de Felício, J. Stat. Mech. (2006) P10002.

[37] R. da Silva, N. A. Alves, and J. R. Drugowich de Felício, Phys. Lett. A 298, 325 (2002).

[38] R. da Silva, N. A. Alves, and J. R. Drugowich de Felício, Phys. Rev. E 66, 026130 (2002).

[39] R. da Silva, H. A. Fernandes, J. R. Drugowich de Felício, and W. Figueiredo, Comp. Phys. Comm. 184, 2371 (2013).

[40] R. da Silva, N. Alves, Jr., and J. R. Drugowich de Felício, Phys. Rev. E 87, 012131 (2013).

[41] R. da Silva, J. R. Drugowich de Felício, and A. S. Martinez, Phys. Rev. E 85, 066707 (2012).

[42] R. da Silva, H. A. Fernandes, and J. R. Drugowich de Felício, Phys. Rev. E 90, 042101 (2014).

[43] E. Arashiro and J. R. Drugowich de Felício, Phys. Rev. E 67, 046123 (2003).

[44] H. A. Fernandes, J. R. Drugowich de Felício, and A. A. Caparica, Phys. Rev. B 72, 054434 (2005).

[45] E. Arashiro, J. R. Drugowich de Felício, and U. H. E. Hansmann, Phys. Rev. E 73, 040902 (2006); J. Chem. Phys. 126, 045107 (2007).

[46] R. da Silva, R. Dickman, and J. R. Drugowich de Felício, Phys. Rev. E 70, 067701 (2004).

[47] H. A. Fernandes, R. da Silva, E. D. Santos, P. F. Gomes, and E. Arashiro, Phys. Rev. E 94, 022129 (2016)

[48] R. da Silva and H. A. Fernandes, J. Stat. Mech. (2015) P06011.

[49] Z. B. Li, U. Ritschel, and B. Zheng, J. Phys. A. 27, L837 (1994).

[50] Z. B. Li, L. Schulke, and B. Zheng, Phys. Rev. E 53, 2940 (1996).

[51] K. Okano, L. Schulke, K. Yamagishi, and B. Zheng, Nucl. Phys. B 485, 727 (1997).

[52] P. Grassberger, Physica A 214, 547 (1995).

[53] C. Chatelain, J. Stat. Mech. (2004) P06006.

[54] R. da Silva and J. R. Drugowich de Felício, Phys. Lett. A 333, 277 (2004).

[55] E. Arashiro, H. A. Fernandes, and J. R. Drugowich de Felício, Physica A 388, 4379 (2009).

[56] Z. B. Li, X. W. Liu, L. Schulke, and B. Zheng, Physica A 245, 485 (1997).

[57] H. Takano, J. Phys. Soc. Jpn. 65, 736 (1996).

[58] C. S. Simões and J. R. Drugowich de Felício, Mod. Phys. Lett. B 15, 487 (2001).

[59] H. K. Janssen and K. Oerding, J. Phys. A: Math. Gen. 27, 715 (1994).

[60] I. A. Hadjiagapiou, A. Malakis, and S. S. Martinos, Physica A 356, 563 (2005).

[61] F. C. Alcaraz and J. R. Drugowich de Felício, J. Phys. A: Math. Gen. 17, L651 (1984). 